\title{
A Road Layout Based Broadcast Mechanism for Urban Vehicular Ad Hoc Networks
}

\author{
Yoonyoung Sung $\mathbb{B}$ and Meejeong Lee $\mathbb{\mathbb { B }}$ \\ Department of Computer Science and Engineering, Ewha Womans University, Daehyun-dong 11-1, Seoul, Republic of Korea \\ Correspondence should be addressed to Meejeong Lee; lmj@ewha.ac.kr
}

Received 18 September 2017; Accepted 2 January 2018; Published 30 January 2018

Academic Editor: Hongwei Wang

Copyright (C) 2018 Yoonyoung Sung and Meejeong Lee. This is an open access article distributed under the Creative Commons Attribution License, which permits unrestricted use, distribution, and reproduction in any medium, provided the original work is properly cited.

\begin{abstract}
Safety services of Vehicular Ad Hoc Network (VANET) require reliable broadcasts. We propose a reliable broadcast mechanism for urban roads called VANET Broadcasting for Urban areas based on Road Layout (VBURL), which tries to minimize the dependency on information that may become inaccurate to maximize the efficiency of broadcast. Specifically, the proposed mechanism takes into account the road layout information accessible from the digital map and only the real-time information obtained from the broadcast messages or beacons. VBURL basically makes the vehicle that is farthest from the current forwarding vehicle take the role of next forwarding vehicle and, if possible, makes an additional broadcast happen at the intersections where the effect of signal attenuation caused by the road side obstacles is low in order to have prompt and reliable dissemination of safety messages towards all roads connected to the intersections. The simulation results verified that VBURL achieves the same high performance as that of the compared legacy schemes in terms of reliability with much higher efficiency. Even though the message reception delay of VBURL is slightly longer than those of compared schemes, it is far less significant to impair the original purpose of safety message.
\end{abstract}

\section{Introduction}

The Vehicular Ad Hoc Network (VANET) consists of vehicles that use mobile communications and enables communication between vehicles or between vehicles and fixed infrastructure along the road. The road condition and traffic information collected through various sensors installed in vehicles and on road side units are transmitted in real time through VANET. The IEEE 802.11p standard and the IEEE 1609 working group defined technologies for Wireless Access in Vehicular Environments (WAVE) [1, 2]. In particular, 802.11p defines the MAC and PHY layers, and 1609.4 defines multichannel operation. For multichannel operation, it is specified to use the control channel (CCH) for safety services, as well as system control such as beacons, and the service channels (SCHs) for general commercial services. The Synchronization Interval (SI) of $100 \mathrm{~ms}$ is divided into an equal length of a $\mathrm{CCH}$ interval and an SCH interval.

VANET enables the provision of services to improve passenger safety and traffic flow through the propagation of information on dangerous situations and the detection of traffic congestion, as well as various services for passenger conveniences and entertainment, such as games, chatting, and data sharing between vehicles [3]. Among these various prospective VANET services, safety related services are expected to be the foremost and representative applications. In particular with the upcoming deployment of autonomous vehicles, the importance of safety service becomes more critical. When a traffic accident occurs or obstacles fall on the road, a safety message is broadcast to encourage deceleration or rerouting to a bypass. Generically, the safety services require reliable broadcasting, and extensive studies have been actively conducted regarding this subject.

Various mechanisms have been proposed to make up for 802.11p for the provision of reliable broadcasts [4-19]. In [4$8]$ the authors proposed extensions of 802.11p to avoid the collisions caused by the hidden terminal. On the other hand [9-16] proposed the acknowledgment schemes for 802.11p to check the reception of broadcast message at the target vehicles and to have the rebroadcast be made if it is needed in order to guarantee the reliable broadcast. In particular, [1719] focus on the reliable broadcast in urban environments 

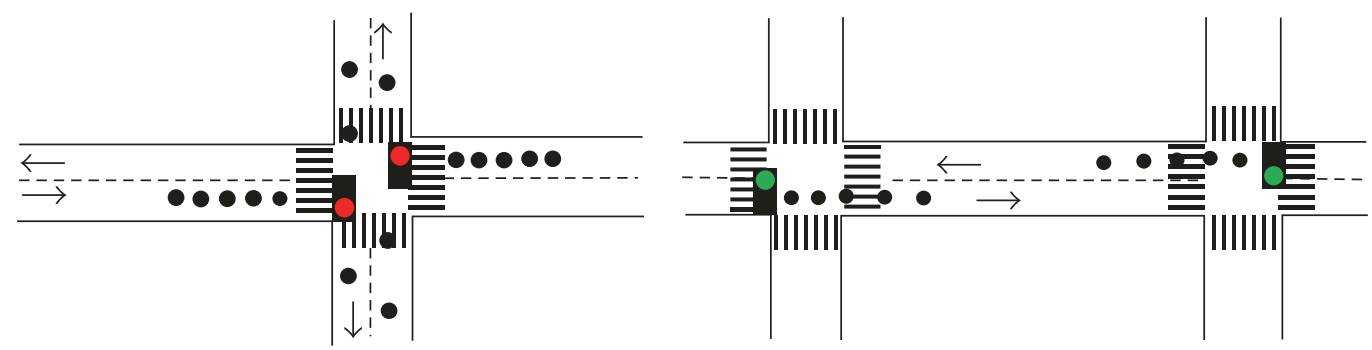

FIGURE 1: Characteristics of urban roads, formation of concentrated vehicle groups due to red light of traffic signals.

and address the problem of broadcast inefficiency in addition to the reliability. Broadcast efficiency is critical in urban environments where the traffic density is relatively high. However, there are some limitations in dealing with the broadcast inefficiency problem at the MAC layer by fully reflecting the characteristics of urban roads.

Differing from [4-19], [20, 21] proposed reliable broadcast mechanisms for VANET to be implemented above the MAC layer, that is, 802.11p. Their common design objectives are to reduce the number of broadcast forwarding attempts by selecting vehicles that can forward the message to the largest possible number of vehicles that have not received the message yet to optimize the broadcast efficiency. Both [20, 21] leverage the location information of neighboring vehicles which has been conveyed though the periodic beacons in order to determine the optimal forwarding vehicles. To this end, the location information is conveyed through the beacons that are periodically exchanged over the $\mathrm{CCH}$ of 802.11p and is kept on hold during a certain holding time.

However, the effect of traffic concentration due to the red light of traffic signals, as shown in Figure 1, is not considered carefully in $[20,21]$. With the traffic concentration, frequent exchanges of beacons may cause congestion in $\mathrm{CCH}$, which leads to the collisions and losses of beacons as well as the safety service messages. On the other hand, increasing the beacon exchanging interval to avoid such congestion also lowers the accuracy of the information provided by the beacons. References $[20,21]$ also implicitly judge the message reception status of neighboring vehicles based on their location information and reflect it to their forwarding vehicle decisions. Around the intersections, though, the implicit judgement on the message reception status frequently turns out to be incorrect due to the signal attenuation by the surrounding buildings. In particular, the inaccuracy of information about the neighboring vehicles may aggravate the broadcast inefficiency more seriously by causing unnecessary broadcast or broadcast message collisions when traffic is heavily concentrated. With the traffic signals and intersections, usually the vehicles with the broadcast message and the vehicles that have not received the message yet meet across one another in groups. With the incorrect information about the neighboring vehicles, wrong and/or the same forwarding priority values are assigned among the neighboring vehicles, resulting in unnecessary broadcasts or broadcast message collisions which wastes the $\mathrm{CCH}$ resources and causes serious efficiency deterioration. Therefore, the legacy schemes that

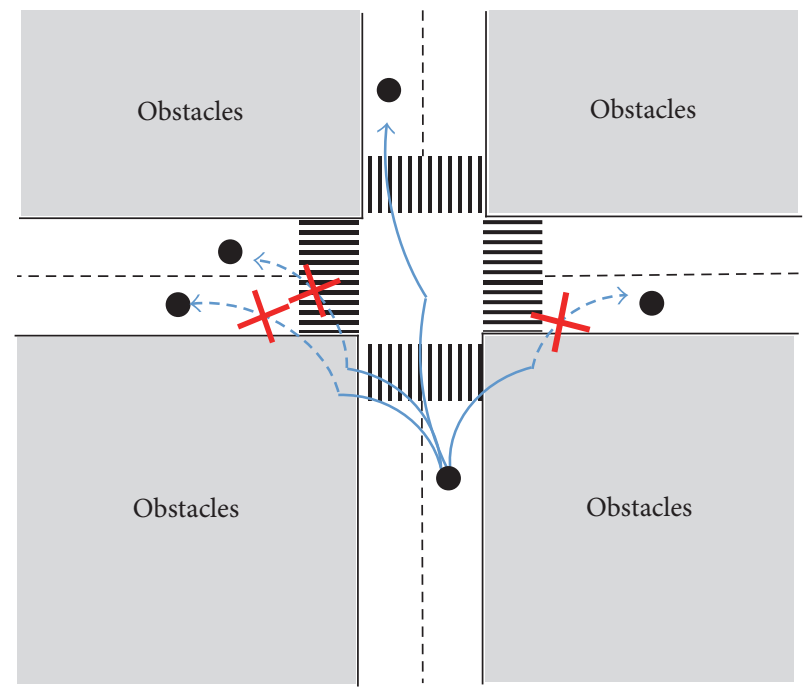

FIGURE 2: Intersection with no vehicle and with significant signal attenuation due to obstacles.

leverages the non-real-time information and the implicit judgement about the message reception status of neighboring vehicles without careful considerations about the characteristics of urban roads need to be reconsidered.

Similar to [20, 21], [22] and a recent work [23] also proposed the broadcast mechanisms for urban roads that is supposed to be implemented above the MAC layer. In [22, 23], differing from $[20,21]$, the road layout information instead of the holding information from beacons is leveraged to minimize the vulnerability to the inaccuracy of information utilized in the forwarding vehicle decisions. The reliability, though, cannot be assured by the works in [22, 23]. For instance, in both $[22,23]$, a vehicle that enters a road segment where the broadcast is just completed cannot receive the broadcast message. In particular, [23] focuses on the broadcast efficiency to eliminate or minimize the redundant broadcast. The delay for broadcast message dissemination, though, may become extraordinarily long in [23] if no vehicle exists at some or many of the intersections and the signal attenuation by surrounding obstacles prohibits the propagation of the broadcast message crossing over those intersections to reach the road segments at the other side of the intersection, as shown in Figure 2.

As the diverse applications of the Intelligent Transportation System (ITS) are expected along with the development of 
VANETs, maximizing the usage efficiency of limited wireless resources is emerging as a critical issue. To this end, studies are conducted on dynamic and flexible utilization of wireless channels based on the real-time usage information [2427]. Under this paradigm, lowering the utilization of $\mathrm{CCH}$ becomes also helpful in enhancing the chances to accommodate additional useful services in $\mathrm{SCH}$ by leveraging the leisurely utilized $\mathrm{CCH}$ resources. Note that $\mathrm{CCH}$ is assigned for the safety message dissemination as well as for the transmission of control messages such as beacons. In order to optimize the efficiency of $\mathrm{CCH}$, therefore, not only the elimination of factors that demand the frequent beacon exchanges but also the maximization of broadcast efficiency of safety message dissemination is necessary.

In this paper, hence, we propose a safety message broadcasting scheme named "VANET Broadcasting for Urban areas based on Road Layout (VBURL)" that enhances the efficiency of safety message broadcast by minimizing the possibility of falsely selecting a forwarding vehicle based on the incorrect information while having little dependency on the beacon interval as well. VBURL is the extension of the work proposed by the authors in [22] to remedy its reliability weakness. Instead of leveraging non-real-time holding information from the periodic beacons or implicit judgement about the message reception status of neighboring vehicles, VBURL takes into account the road layout information accessible from the digital map similar to [22, 23], which is common and available already through the Digital Multimedia Broadcasting (DMB) networks. The performance of VBURL is, therefore, much less vulnerable to the beacon cycles as well as to the usual urban road characteristics such as high traffic density, frequent traffic signals and intersections, and traffic concentration.

Note that the urban roads consist of intersections and the roads interconnecting them. In VBURL, different forwarding strategies are applied depending on whether there is an intersection within the transmission range of a vehicle that receives the broadcast message and is supposed to determine whether to take the role of message forwarding. It is assumed that the signal attenuation caused by obstacles along the road sides is not significant on the straight roads between intersections, and thus, VBURL simply makes the vehicle that is farthest from the current forwarding vehicle perform the rebroadcast on the straight roads for rapid and efficient broadcasting. Meanwhile, for the message dissemination around the intersections, VBURL makes an additional broadcast happen at the intersections in addition to the one made by the farthest vehicle as long as there exists a vehicle hearing the broadcast at the intersection or else at least there exist one or more vehicles that are moving toward the intersection with that intersection within their transmission range. Since the effect of signal attenuation caused by the road side obstacles is low inside of the intersections, it enables the prompt and reliable dissemination of safety message toward all roads connected to the intersections.

This paper is organized as follows. After the introduction in Section 1, Section 2 examines the related studies. VBURL is explained in detail in Section 3. Section 4 describes the simulation to compare the performance of VBURL with that of two representative legacy schemes and analyzes the results of simulation. Finally, Section 5 provides a conclusion.

\section{Related Work}

In this section, existing studies that implement the efficiency of reliable broadcasting on urban roads on top of the plain 802.11p, similar to VBURL, are examined [20, 21, 23]. These schemes commonly use the Connected Dominating Sets (CDS) [28] as the virtual backbone network for broadcasting. If a correct CDS can be set up, the broadcast dissemination to entire network is possible solely by the broadcast relays of vehicles included in the CDS. These schemes, hence, strive to suppress unnecessary transmissions by non-CDS vehicles in order to maximize the efficiency. References [20,21] leverage the holding information obtained from the beacons such as the location of neighboring vehicles to determine the CDS, whereas the road layout information is utilized in [23].

2.1. Acknowledged Parameter-Less Broadcast in Static to High Mobile (ABSM). ABSM uses the Neighbor Elimination Scheme (NES) as proposed in $[28,29]$ as well as CDS vehicle information to determine the broadcast-forwarding vehicle [20]. In ABSM, the vehicles implicitly decide the message reception status of their neighboring vehicles by considering the distance between the forwarding vehicle and the neighboring vehicle leveraging the location information of neighboring vehicle obtained from the beacon. Each vehicle manages neighboring vehicle lists of $R$ and $N$, and the neighboring vehicles that are believed to have received the message are compiled in list $R$ and the other vehicles are placed on the list $N$. Upon receiving the message, each vehicle sets the broadcast timer for deciding the next forwarding vehicle. In ABSM, the broadcast timer value, which determines the forwarding vehicle, is set combining two factors: (1) whether the vehicle is in the CDS, and (2) the number of neighboring vehicles that have not received the message, that is, $|N|$. A CDS vehicle sets the timer to be inversely proportional to the number of neighboring vehicles that have not received the message. For the timer value of non-CDS vehicles, a constant value of 'maximum waiting time' is added to that of CDS vehicle so that the timer of CDS vehicle always expires first before that of non-CDS vehicle when they have the same number of neighboring vehicles that have not received message. The vehicle whose timer has expired performs a broadcast and the other vehicles that receive the duplicate broadcast release their broadcast timer.

Furthermore, message reception is indicated in the beacon so that, when a new vehicle with no message appears as a neighboring vehicle, it can be detected and the message can be sent to the new neighbor. ABSM updates the lists $R$ and $N$ through the broadcast message reception and the beacon exchange. Broadcast is repeated until no neighboring vehicles exist in the list $N$ so that the reliability of message transmission to the entire network can be guaranteed.

In ABSM, not only the CDS virtual backbone network set up but also the broadcast timer setting is determined based on the holding information conveyed by the beacons. In order for ABSM to operate efficiently, the real-time locations of 


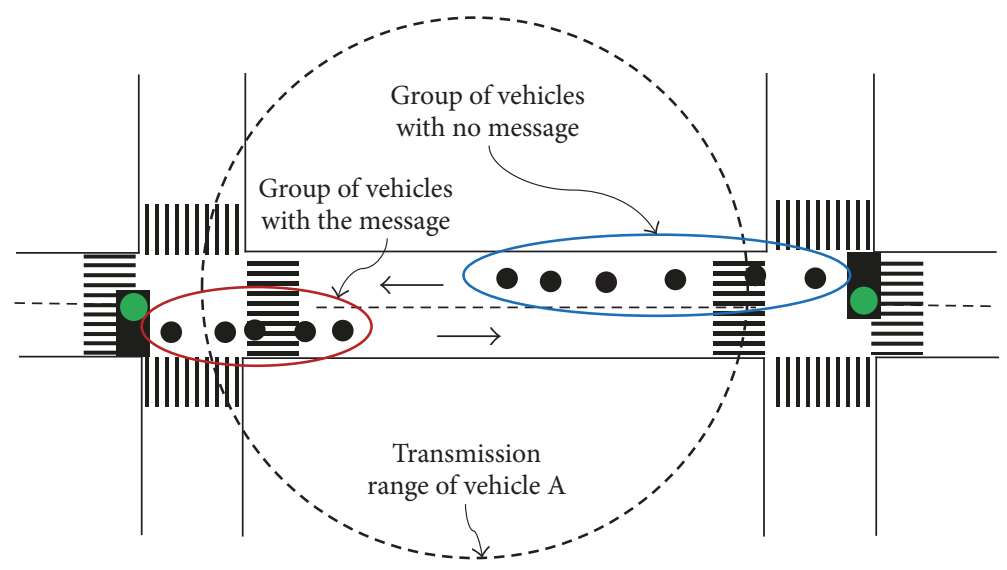

FIGURE 3: Example of a general situation where vehicles with no message are discovered through a beacon.

neighboring vehicles and positional relationships must be accurately identified. On urban roads, which have frequent intersections and high vehicle densities, however, the beacon may not be received properly due to signal attenuation or a collision, and thus, the locations of neighboring vehicles may not be accurately identified or actual neighbors may not be recognized as neighbors (or vice versa), lowering the broadcast performance of ABSM.

Especially, with the traffic concentration at the red traffic light, it is more likely that there exist multiple vehicles with the same number of neighboring vehicles that have not received the broadcast message, resulting in a broadcast collision. Broadcast collision degrades the performance of ABSM by causing additional broadcasts. In particular, many such broadcast collisions can occur in a series of attempted broadcasts when vehicles with no message are discovered by a beacon. Figure 3 shows the situation where vehicles with no message are discovered by a beacon. Due to the traffic concentration phenomenon of urban roads, vehicles with the message and vehicles with no message frequently approach each other in groups. Note that the discovery of neighbors and the confirmation of message possession can be verified only through beacons, and the beacons of different vehicles are not synchronized. When the first beacon from a vehicle in the group with no message is generated, multiple vehicles in the group with the message recognize it simultaneously and set the broadcast waiting timer accordingly. In this case, the same waiting time is set for all the vehicles with the same CDS status, and this results in simultaneous broadcasts. In case the broadcast waiting time of ABSM is shorter than the beacon cycle, a "broadcast and then collision" incident occurs repeatedly whenever each vehicle in the group with no message generates a beacon.

Furthermore, ABSM may incorrectly judge the message reception status of neighboring vehicles. ABSM implicitly determines the message reception status of neighbors by considering the distance between a neighbor and a messageforwarding vehicle without considering the signal attenuation by obstacles. This may, however, turn out to be a false guess. In particular, as shown in Figure 4, the reception status of neighbors cannot be accurately guessed near an intersection. The broadcast of the forwarding vehicle may not be delivered to the vehicles on roads $\mathrm{R} 1$ and $\mathrm{R} 2$, which are close to the intersection, due to signal attenuation. However, vehicle A thinks that its neighboring vehicles on roads $\mathrm{R} 1$ and $\mathrm{R} 2$ have received the message by only considering the distance between the neighbors and a message-forwarding vehicle. This incorrect judgement affects the determination of the optimum forwarding vehicle.

2.2. Receiver Consensus ( $\mathrm{ReC}$ ). $\mathrm{ReC}$ is proposed to improve the speed of message delivery as well as the broadcast efficiency [21]. As with ABSM, ReC also constructs a CDS virtual backbone network for broadcast delivery. While ABSM determines the priority of forwarding vehicles according to the combined factors, that is, the number of neighboring vehicles that have not received the message and whether the vehicle is a CDS vehicle or not, ReC definitively gives higher priorities to CDS vehicles than to non-CDS vehicles. The broadcast priorities among the vehicles with the same CDS status are determined based on the ideal location, which is the average location of neighboring vehicles that have not received the broadcast message. The first time slot is assigned to the first priority vehicle for immediate broadcast with no delay. Furthermore, the $n$th time slot is assigned to the vehicle of $n$th priority, and the broadcast is carried out if no broadcast is heard in the previous time slots before the expiration of its own broadcast timer. Since CDS status of neighboring vehicle is also reflected in the decision of forwarding vehicle in $\mathrm{ReC}$, CDS information is indicated in the beacon together with the location of vehicle.

ReC manages neighbors with three lists by adding list $P$ to lists $R$ and $N$, which are also used in ABSM. ReC is similar to ABSM in that neighbors are classified (lists $P$ and $N$ ) by judging message reception status based on the locations of neighbors informed by the beacons and the location of the current forwarding vehicle. A vehicle in $P$ is moved to $R$ only when message reception is explicitly confirmed by a beacon. For each vehicle, the beacon cycle is divided by $|R|+|P|$ time slots. The forwarding vehicle that has performed broadcast moves its neighbors in list $N$ to list $P$, and the vehicles that 


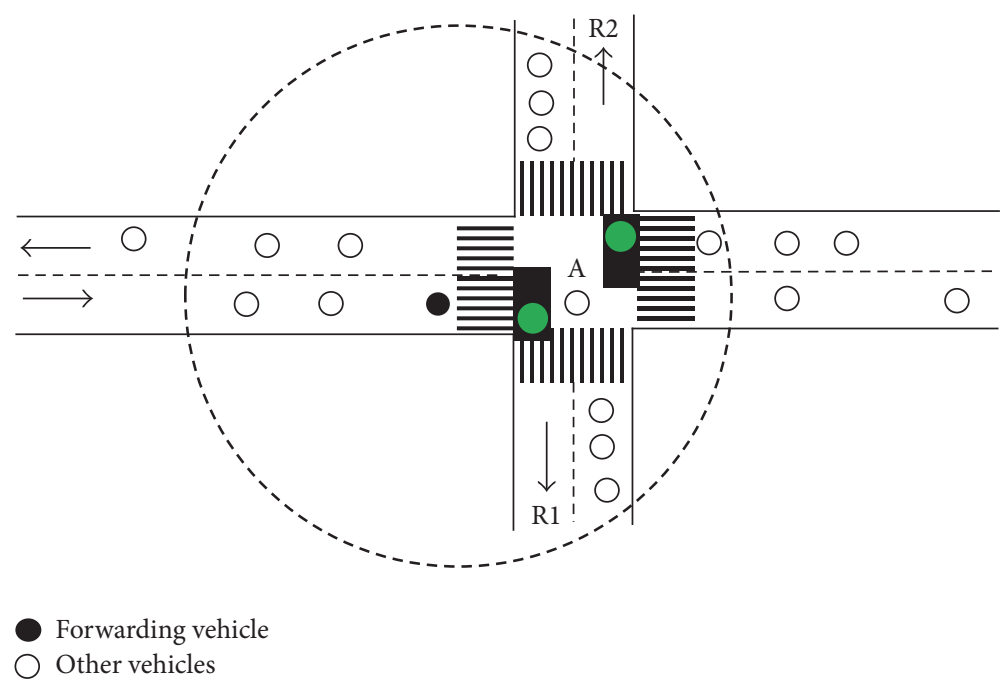

FIgURE 4: Example of a broadcast near an intersection.

have received message repeat this process until there is no vehicle in list $N$, thus ensuring message dissemination to the entire network.

As with ABSM, however, the inaccuracy of beacon information due to the signal attenuation around the intersection or beacon collisions as well as non-real-time characteristics of holding information may result in suboptimal selection of forwarding vehicle.

2.3. Broadcast Protocol with Road Network Topology (BRNT). Differing from ABSM and ReC, BRNT configures a CDS consisting of the road segments connecting the intersections instead of the individual vehicles [23]. Upon receiving a broadcast message, the broadcast timer of a vehicle is set according to where they are located so that the vehicles located at the intersections are assigned with the highest forwarding priority among the vehicles that receive the broadcast message, then the vehicles on the CDS road segments, and finally the ones on the other road segments. Specifically, the broadcast timer of the vehicles on a higher forwarding priority road segments is set with a shorter timer expiration value, and a vehicle forwards the broadcast message upon the expiration of its timer while the vehicles receiving the duplicate message cancel their message forwarding. The broadcast timer is further tuned so that the farther vehicle from the current forwarding vehicle is assigned with the higher forwarding priority on the same road segment.

If there exist one or more vehicles at every intersection or the signal attenuation at intersections is not significant so that all CDS road segments are connected by the intersections, BRNT could work optimally in terms of both delay and efficiency since the message is disseminated via the CDS road segments which have the shortest waiting time for forwarding, with no duplicate transmissions. As the ratio of intersections with no vehicle or with nonnegligible signal attenuation gets higher, though, the broadcast dissemination delay could get very long since not only some of the road segments connected to those intersection can only receive the message via a detouring path but also the broadcast message may have to be disseminated via the non-CDS road segments, which requires longer forwarding delay than the CDS road segments, due to the incomplete connectivity of CDS road segments. Note that chance of having broadcast at the intersection is higher in the proposed VBURL than in BRNT since intersection broadcast cannot happen in BRNT if there is no vehicle at the intersection at the point of broadcast message reception, whereas VBURL may have the vehicle moving toward the intersection perform the intersection broadcast even in the case where no vehicle exists at the intersection at the point of broadcast message reception. Furthermore, no mechanism is provided to ensure the delivery of broadcast message to a vehicle that newly appears or missed the broadcast due to some signal interference on the road segment where the message broadcast has been completed once.

\section{VANET Broadcasting for Urban Areas Based on Road Layout}

Safety service aims to alert the occurrence of a traffic accident or the falling of obstacles on the road through broadcasts to all vehicles near the point of accident so that they can decelerate or reroute to a bypass. The purpose of the proposed VBURL is to improve the efficiency while ensuring the reliability of safety message broadcasting by considering the environmental characteristics of urban roads such as a high vehicle density, as well as frequent intersections and traffic signals. VBURL attempts to take appropriate broadcast dissemination in consideration of the road layout without depending on neighboring vehicle information which is identified through a beacon. On straight roads between intersections, where the effect of signal attenuation by obstacles is low, it allows the vehicle located farthest from the current forwarding vehicle perform the next broadcast forwarding in order to deliver the message to the largest possible number of vehicles similar to the Contention Based Forwarding (CBF) [30]. Meanwhile, to facilitate the message dissemination around the intersections, VBURL makes an additional broadcast happen 


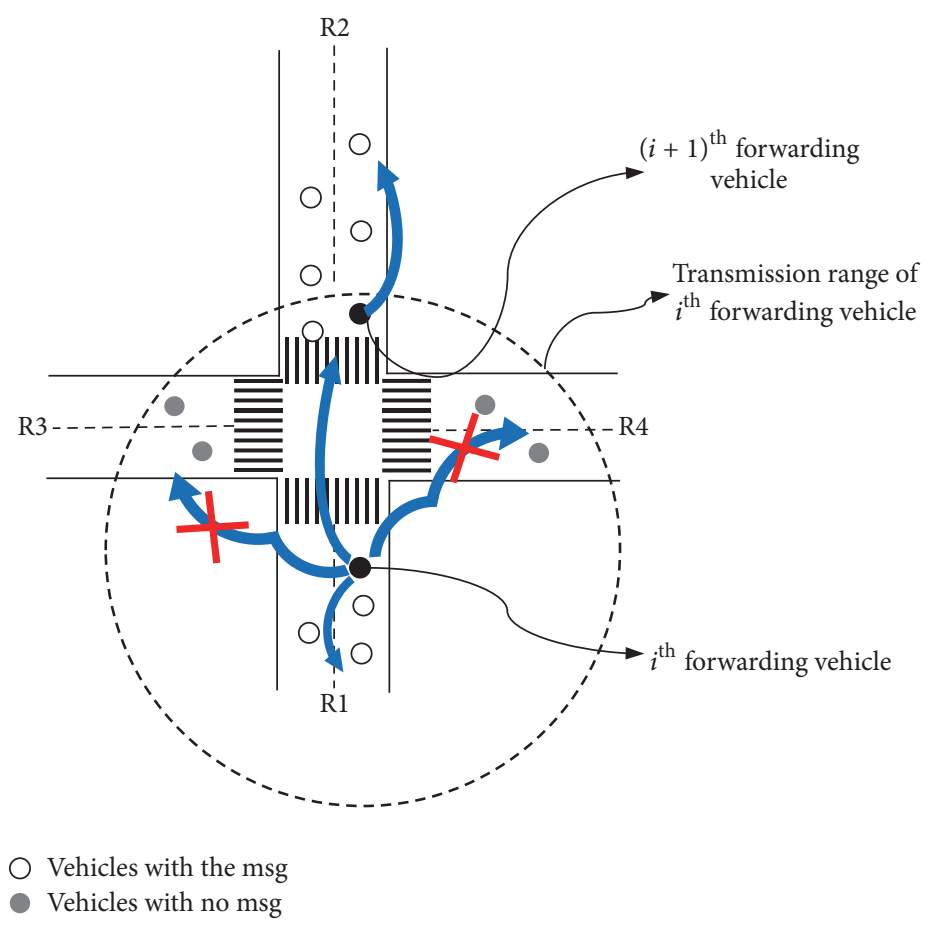

Figure 5: Broadcasting near an intersection.

at the intersections in addition to the one made by the farthest vehicle as long as there exists a vehicle hearing the broadcast at the intersection or else there exist one or more vehicles that are moving toward the intersection with that intersection within their transmission range. Since the effect of signal attenuation caused by the road side obstacles is low inside of the intersections, it facilitates the prompt and reliable dissemination of safety message toward all roads connected to the intersections. Furthermore, in order to achieve reliability, a rebroadcast is performed if broadcast dissemination of the next forwarding vehicle is not heard or a neighboring vehicle that has not received the message is discovered by a beacon. In Section 3.1, the safety message broadcasting method of VBURL is explained. The rebroadcasting method for the reliability in safety message dissemination is explained in Section 3.2 .

3.1. Broadcasting of Safety Message. When a vehicle receives a new safety message that is required to be broadcast, it determines the forwarding method depending on the existence of an intersection within its transmission range. First, the forwarding method of a vehicle that has no intersection within its transmission range is described. The forwarding method when there is an intersection within the transmission range is then described.

3.1.1. Broadcasting with No Intersection. The vehicle that has received a new safety message saves it in the broadcast message buffer and maintains it until the expiration of message life time. In addition, it uses the Forwarding Timer (FT) to determine the next forwarding vehicle for continuous dissemination of the message. Similar to CBF, every vehicle receiving the safety message sets its FT in inverse proportion to its distance from the current forwarding vehicle using the location information of forwarding vehicle conveyed in the message, and the vehicle whose timer expires before hearing others' broadcast performs the broadcast. As a result, the farthest vehicle from the current forwarding vehicle takes the role of message-forwarding vehicle. On a straight road where the effect of obstacles is relatively little, broadcasting by the vehicle that is farthest from the message-forwarding vehicle potentially disseminates the message to the largest possible number of vehicles that have not received the message.

If the received safety message is already stored in the buffer, it will be labeled as a duplicate message. When a vehicle receives a duplicate of the message for which the FT is running, it means that another vehicle already has taken the role of the next forwarding vehicle. In this case, the FT is released to avoid unnecessary duplicate broadcasts.

3.1.2. Broadcasting with an Intersection. Near an intersection, due to the effect of obstacles such as buildings around the intersection, there can be roads where message dissemination is impossible through the simple broadcasting by a vehicle whose FT expires first. For instance, the vehicles on roads R3 and R4 in Figure 5 cannot receive the message forwarded by the vehicles that are selected according to the FT expiration because of the signal attenuation due to the buildings around the intersection. On the other hand, as shown in Figure 6, the center of an intersection is least vulnerable to the signal attenuation by surrounding obstacles. In this paper, it is defined as the "intersection area." To facilitate prompt and reliable broadcast message dissemination to all roads adjacent to an intersection, hence, VBURL maximizes the chance of broadcast occurring within the intersection area by having not only the vehicle in the intersection area but also the vehicle 


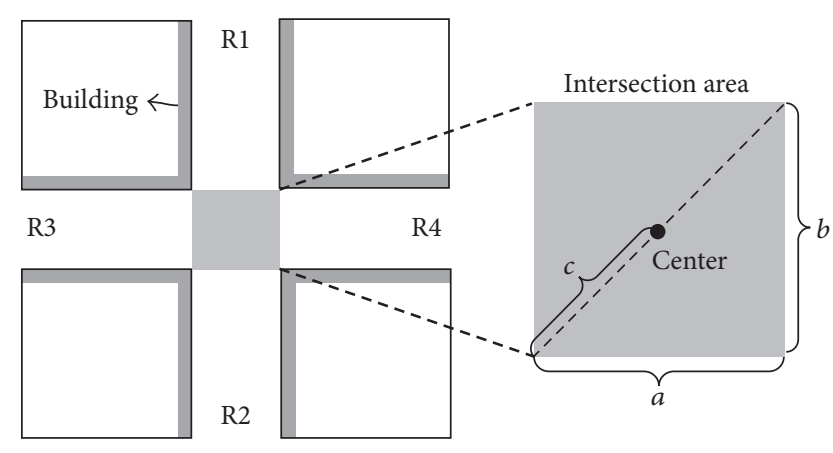

FIGURE 6: Intersection area.

moving toward the intersection, in case there is no vehicle in the area at the point of broadcast message reception, perform the intersection area broadcast forwarding. Specifically, VBURL makes the first vehicle that arrives at the intersection perform the intersection area broadcast forwarding in case there is no vehicle at the intersection at the point of broadcast message reception but there exist one or more vehicles moving toward the intersection with that intersection within their transmission range.

In order to do this, the vehicles that have the intersection in their transmission range (groups (1) and (2) in Figure 7) and the vehicles that are already located within the intersection area (group (3) in Figure 7) set the Intersection Forwarding Timer (IFT) according to (1) in addition to the FT. The vehicles in the intersection area, group (3), set their IFT in proportion to their distance from the center of intersection, so that the vehicle closer to the center of intersection where the effect of signal attenuation by surrounding buildings is the least will take the role of broadcasting before others. On the other hand, the vehicles moving toward the intersection, such as groups (1) and (2), set their IFT to the maximum value that is large enough not to expire until they reach the intersection $\left(\right.$ IFT $\left._{\max }\right)$. Upon the arrival at the intersection, if they have not heard the broadcast from the intersection area yet, they reset their IFT to IFT time_out. Consequently, when there are vehicles in the intersection area, the vehicle that is closest to the center of the intersection area takes charge of broadcasting within the intersection area, whereas, if there is no vehicle in the intersection area, the vehicle that arrives at the intersection first takes charge of it.

The goal of IFT is to facilitate the dissemination of broadcast message to all roads adjacent to the intersection. Thus, it is released only when a duplicate broadcast from the intersection area is heard. The FT and IFT may be running simultaneously at a vehicle for a specific safety message, and whichever that expires first makes the broadcast be performed. If the FT expires first and the vehicle is in the intersection area, it means that broadcast is already occurring in the intersection area due to the FT expiration, and the IFT is released to prevent an unnecessary duplicate broadcast. On the other hand, if the vehicle is still on its way to the intersection upon the broadcasting caused by the FT expiration, broadcast within the intersection area is still necessary and the running IFT is maintained. If the FT is running in the vehicle when the IFT expires, the FT is always released to prevent unnecessary duplicate broadcasts:

$$
\mathrm{IFT}= \begin{cases}\mathrm{IFT}_{\text {time_out }}=\operatorname{MaxWT} \times \frac{D_{\text {vehicle_to_center }},}{c}, & \text { if intersection vehicle } \\ \mathrm{IFT}_{\max }, & \text { if intersection within TR and approacing intersetion } .\end{cases}
$$

(i) IFT $_{\max }$ is maximum timer value that is large enough not to expire until the vehicle enters the intersection;

(ii) MaxWT is maximum waiting time;

(iii) $D_{\text {vehicle_to_center }}$ is distance from the center of intersection to the vehicle;

(iv) $c$ is half of the diagonal length of the intersection area (see Figure 6).

3.2. Rebroadcast of Safety Messages. For reliable broadcasting, the safety message must be rebroadcast in case the safety message fails to be disseminated to the next forwarding vehicle. For this purpose, it must be possible for the current forwarding vehicle to confirm the reception of a safety message by the next forwarding vehicle. There are largely two methods of confirming the receipt of a safety message: explicit acknowledgment, which requires the exchange of a control message, and implicit acknowledgment, which regards the broadcast of safety message by the next forwarding vehicle as the reception confirmation message and considers that all vehicles located between itself and the next forwarding vehicle have received the safety message [9-13]. In VBURL, the implicit acknowledgment is deployed for the efficient use of limited wireless resources considering the VANET environments, similarly to the method used in other studies $[9,12$, 13]. In general, when the implicit acknowledgment method is used, if the broadcast of the next forwarding vehicle is not heard within a certain amount of time after broadcasting, rebroadcasts are performed repeatedly for a maximum count. In addition, if the vehicle with an effective safety message discovers a neighbor that has not received the message after the broadcast has been completed, it makes an additional broadcast to improve reliability.

3.2.1. Rebroadcast of a Forwarding Vehicle. When the vehicle that has broadcast a safety message receives it redundantly from another vehicle, it assumes that successful progress of continuous message dissemination is made by next forwarding vehicles. Otherwise, the vehicle determines that the dissemination of broadcast has stopped and performs a rebroadcast. For this purpose, every forwarding vehicle sets the 


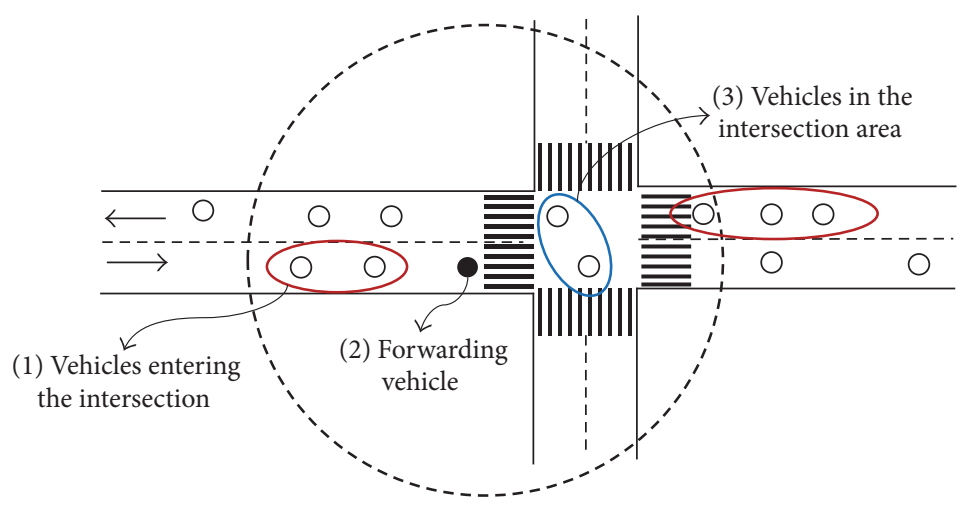

FIGURE 7: Target vehicles of IFT setting for broadcasting in the intersection area.

Rebroadcast Timer (RT) after broadcasting a message. In the study [9], if a vehicle that has performed broadcast receives a broadcast from the next forwarding vehicle, it assumes that all neighboring vehicles between the two forwarding vehicles have received the message. However, this may not be a valid assumption if there are roads where broadcast dissemination is not smooth due to signal attenuation by obstacles. In particular, around the intersection, receiving a broadcast from the next forwarding vehicle that comes from a certain road may not mean successful progress of broadcast dissemination toward every road adjacent to the intersection. Consequently, in VBURL, the vehicle that has performed broadcast in the intersection area sets the Intersection Rebroadcast Timer (IRT) to verify that the message has been delivered to all roads adjacent to the intersection while the forwarding vehicle that has performed broadcast with no intersection within its transmission range sets the RT. Receiving a duplicate message for which the RT is running means that the next forwarding vehicle has successfully received the message that the vehicle has broadcast. Thus, the vehicle considers that its responsibility for message dissemination has been transferred to the next forwarding vehicle and releases the RT. On the other hand, the IRT is released after receiving the duplicate message from all roads adjacent to the intersection where neighbor vehicles exist, because the purpose of IRT is to confirm that the message has been disseminated to all roads adjacent to the intersection. For the purpose of figuring out the roads where neighboring vehicles exist and for this purpose only, VBURL leverages the holding information which has been obtained from the periodic beacons.

To avoid early expiration, RT and IRT are defined according to (2) considering the time over which the safety message has been disseminated to the transmission range and the delay until the safety message returns by broadcasting after the broadcast waiting time of the next forwarding vehicle. RT or IRT expires when there is a road where message dissemination has not been confirmed. Therefore, when RT or IRT expires, the forwarding vehicle rebroadcasts the same message and sets the timer again to wait until message reception is confirmed from all roads to which the broadcast must be disseminated. These timers can be reset for the maximum number set by the system so as to repeat the rebroadcast process as needed:

$$
\mathrm{RT}_{\text {timeout }}=\mathrm{MaxWT}+2 \times \mathrm{MaxPT} \text {. }
$$

(i) MaxWT is maximum waiting time;

(ii) MaxPT is maximum dissemination delay of the broadcast message, which is the time until the message is disseminated to the transmission range.

3.2.2. Rebroadcast for the New Neighboring Vehicles with No Message. Neighboring vehicles that have not received a broadcast of the safety message may appear for various reasons, such as collision, radio interference, and the entrance of a new vehicle. In this case, an additional broadcast must be performed to deliver the safety message to the vehicle with no message. The Neighbor Forwarding Timer (NFT) is used for this purpose. The NFT is set when none of FT, IFT, RT, and IRT is running for a valid safety message, and the message is not specified in the received message list in the beacon of a neighbor. The purpose of NFT is to avoid the collision of broadcasts or unnecessary broadcasts by deciding the optimum vehicle that will take the broadcasting role when multiple vehicles with the message discover a vehicle with no message simultaneously in a geographically close area. For this purpose, among the vehicles that have the message and have received a beacon from a vehicle with no message, VBURL makes the vehicle closest to the vehicle with no message perform the broadcast of safety message. Considering that usually the vehicles with no message and the vehicles with the message encounter each other in groups on urban roads due to the traffic signals as shown in Figure 3, if a vehicle closest to the vehicle with no message takes charge of message delivery, it can deliver the message to more vehicles with no message through one broadcast. Specifically, the values of the NFT are set by (3). The distance among the vehicles with the message may be quite small due to vehicle concentration upon the wait traffic signal. To prevent unnecessary broadcasts, VBURL makes the difference in NFT 


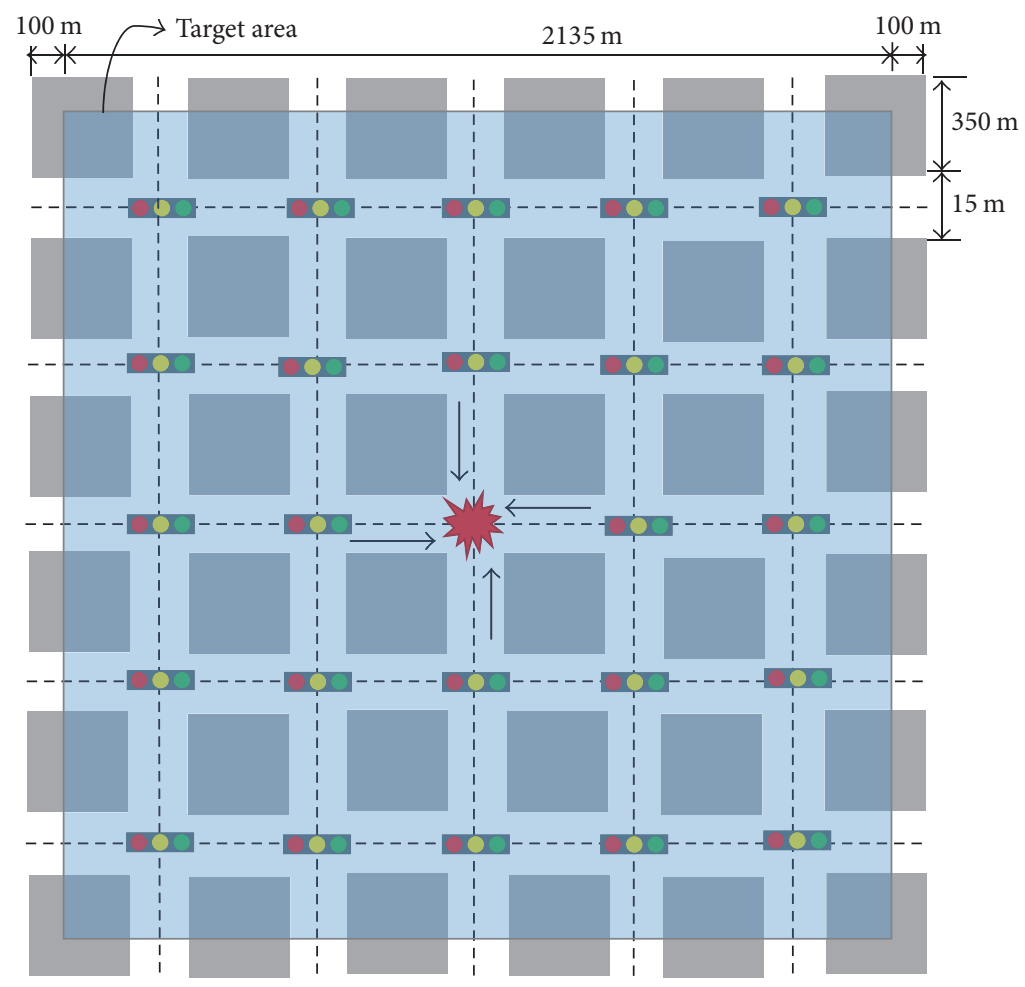

FIGURE 8: Urban road layout for simulation.

values set by vehicles with the message be greater than the propagation delay by increasing MaxWT $n$ times in NFT value calculation. As with the FT, unnecessary duplicate broadcasts are avoided by releasing the NFT when a vehicle running the NFT receives a duplicate broadcast, that is, when the message has been delivered by another forwarding vehicle. NFT may also be released if it is confirmed through the beacons that have been received during the running of NFT that all neighbors have received the message:

$$
\mathrm{NFT}_{\text {timeout }}=n \times \operatorname{MaxWT} \times \frac{D}{\mathrm{TR}} .
$$

(i) MaxWT is maximum waiting time;

(ii) $D$ is distance between a vehicle with a message and a vehicle with no message;

(iii) $\mathrm{TR}$ is transmission range.

The broadcasting and rebroadcasting mechanisms of VBURL are illustrated in pseudocodes in Algorithm 1.

\section{Performance Evaluation}

A simulation is conducted using OPNET to compare the performance of VBURL with ABSM and ReC, which are the legacy schemes implementing the reliable broadcast for urban roads on top of IEEE 802.11p. The simulation environments are first explained in Section 4.1. In Section 4.2, the numerical results of simulation are then presented.
4.1. Simulation Environments. The urban roads used in the experiment are bidirectional one-lane roads where 25 intersections are located at intervals of $350 \mathrm{~m}$ as shown in Figure 8. It is assumed that $10-20 \mathrm{~m}$ high buildings are located along the road and the signal attenuation by the surrounding buildings is implemented using the knife-edge model [31].

At the beginning of the simulation, vehicles running at random speeds start one by one at fixed intervals at each of the 20 entrances in the simulation network roads and are directed to run straight along the road. The vehicle arriving at the end of the road exits the simulation network, and a new vehicle instead starts on the lane of the opposite direction to maintain a constant vehicle density on the simulation network roads. Each intersection has signal lights, and the vehicles repetitively drive/wait according to the signals. All of the signal lights on the east-west roads turn to driving (waiting) signals simultaneously, and all of the signal lights on the south-north roads turn to waiting (driving) signal simultaneously. The vehicles are set to stop at $4 \mathrm{~m}$ intervals at the waiting signal of intersections considering the average body length of vehicles.

As shown in Figure 8, the accident point is at the center of the simulation network, and it is assumed that the safety message is first broadcast from the accident point. The eastwest and south-north roads, excluding $100 \mathrm{~m}$ on each edge, are set as the target area (shaded area in Figure 8) to which the safety message must be broadcast. Furthermore, the vehicles in the target area when the safety message is broadcast for the first time are assumed to be the target vehicles that are considered in the message reception ratio. The safety message 


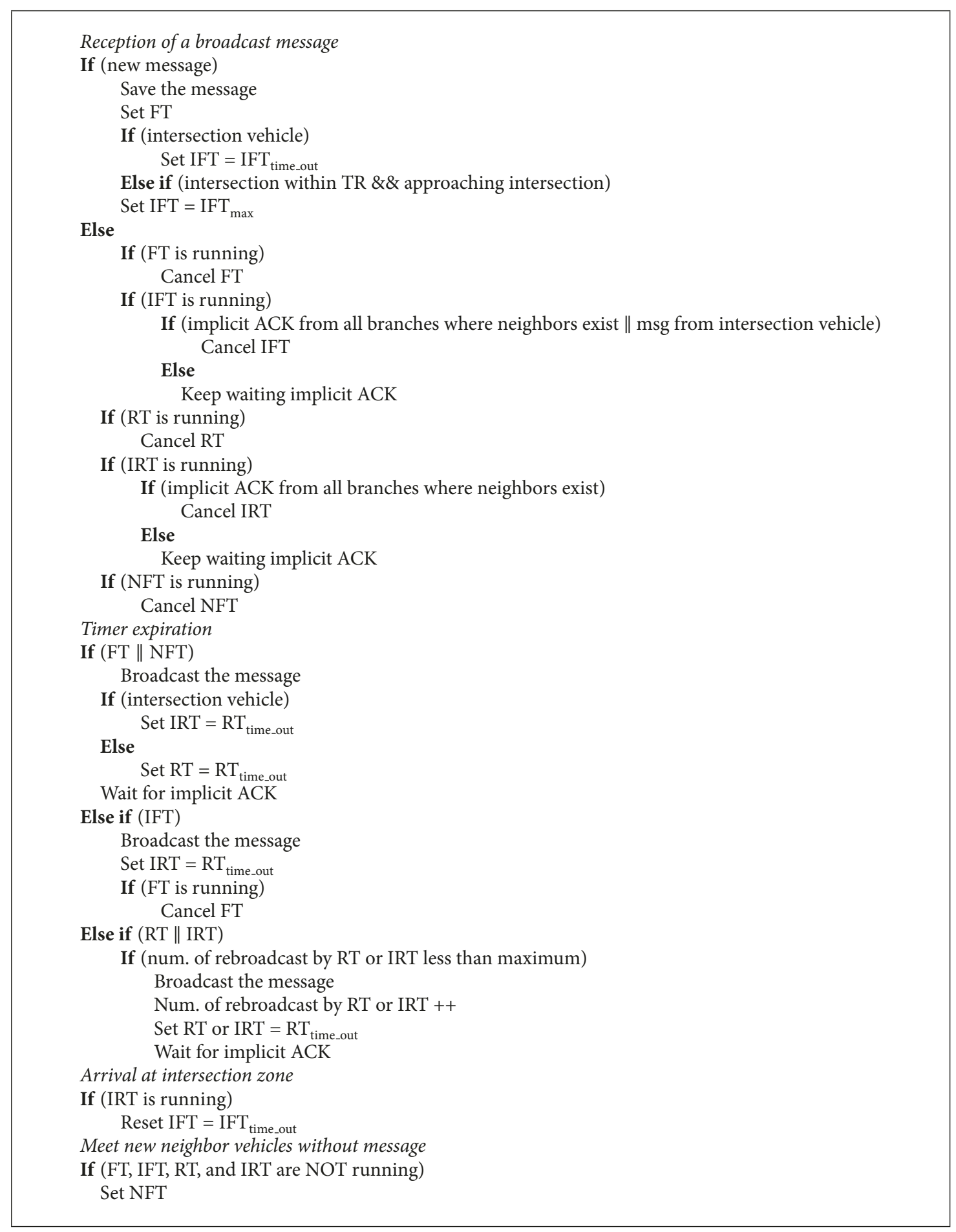

Algorithm 1: Pseudocode of VBURL.

size is 100 bytes, and the life time of the safety message is set to 100 seconds.

Both ABSM and $\mathrm{ReC}$ use the location information of neighboring vehicles transmitted through beacons, and when the beacon cycle increases, the accuracy of neighboring vehicles' location information decreases. Furthermore, all of the three compared schemes discover the neighboring vehicles with no message by exchanging the received safety message identifier through beacons. Thus, when the beacon cycle is short, the broadcast dissemination attempts for the vehicles with no message are made frequently. In this experiment, therefore, beacon exchange cycle, which is an important parameter that can affect the performance, is increased to 0.5 , $1.0,3.0,5.0,7.0$, and 10.0 seconds. 
TABLE 1: Simulation Parameters.

\begin{tabular}{lc}
\hline Simulation parameters & Values \\
\hline Transmit power & $0.005 \mathrm{~W}$ \\
\hline Min. frequency & $5885 \mathrm{MHz}$ \\
\hline Data rate & $6 \mathrm{Mbps}$ \\
\hline Channel bandwidth & $10 \mathrm{MHz}$ \\
\hline Transmission range & $300 \mathrm{~m}$ \\
\hline Network dimensions & $\begin{array}{c}\text { Target area, } 2,135 \mathrm{~m} \times 2,135 \mathrm{~m}, 1 \text { lane } \\
\text { in } 2 \text { directions }\end{array}$ \\
\hline Vehicle velocity & $\begin{array}{c}20-30 \mathrm{~km} / \mathrm{h}(\text { congestion scenario) } \\
40-50 \mathrm{~km} / \mathrm{h}(\text { smooth scenario) }\end{array}$ \\
\hline $\begin{array}{c}4000 \text { vehicles, } 86.4 \text { vehicles } / \mathrm{km} \\
(\text { congestion scenario })\end{array}$ \\
Vehicle density & $\begin{array}{c}2000 \text { vehicles, } 43.2 \text { vehicles } / \mathrm{km} \\
(\text { smooth scenario })\end{array}$ \\
\hline Beacon interval & $(0.5,1.0,3.0,5.0,7.0,10.0)$ seconds \\
\hline Beacon hold time & $(1.5,3.0,9.0,15.0,21.0,30.0)$ seconds \\
\hline
\end{tabular}

The driving speed on urban roads is generally slower than on the express or freeways due to a high vehicle density and frequent intersections. Furthermore, it is usual to have higher traffic density during the commuting hours due to a large number of inflowing vehicles. In this experiment, two scenarios of traffic flow are assumed: a case of rush hours, such as commuting hours, during which vehicles are congested (hereinafter referred to as the "congestion scenario"), and a case of noncommuting hours, during which the traffic flow is relatively smooth (hereinafter referred to as the "smooth scenario"). For the congestion scenario, longer traffic signal cycles are set to reflect the reality. Specifically, for the congestion scenario, 200 vehicles running at random speeds between 20 and $30 \mathrm{~km} / \mathrm{h}$ start at $1.5 \mathrm{sec}$ intervals at each road entrance (a total of 4000 vehicles, i.e., traffic density of 86.4 vehicles $/ \mathrm{km}$ ), and the waiting/driving signal duration is set to 25 seconds. For the smooth scenario, 100 vehicles running at random speeds between 40 and $50 \mathrm{~km} / \mathrm{h}$ start at $2 \mathrm{sec}$ intervals at each road entrance (a total of 2000 vehicles, i.e., traffic density of 43.2 vehicles $/ \mathrm{km}$ ), and the signal changes at intervals of 15 seconds.

In this experiment, the MaxWT value is set to $0.1 \mathrm{sec}$, which is used to set the $\mathrm{FT}\left(\mathrm{FT}_{\text {timeout }}=\operatorname{MaxWT} \times(1-D / \mathrm{TR})\right)$, IFT, RT, and IRT in VBURL, and the MaxPT used to set the $\mathrm{RT}$ and IRT is set to $0.003 \mathrm{sec}$. For the NFT, $n$ is set to 10 for the maximum waiting time of $1 \mathrm{sec}$. The parameter for broadcast in ABSM is set to $W=0.25 \mathrm{sec}$ according to [20]. The maximum waiting time used to set the waiting time in $\mathrm{ReC}$ is set as the beacon cycle according to [21]. Table 1 outlines the major simulation parameters used in this experiment and their values.

The performance measurement values collected for performance evaluation are as follows. All the measurement values are averages of the measurements for 10 broadcasts.

(1) Message reception ratio: this is the ratio of vehicles that have received the message before expiration of the life time of the message among all the target vehicles. It is used to evaluate the reliability of a broadcast scheme.

(2) Number of receiving vehicles per broadcast: this is the number of receiving vehicles per broadcast forwarding. It is used to evaluate the efficiency of a broadcast scheme.

(3) End to end delay: this is the time elapsed until a target vehicle receives a message after the message is first generated. It is used to evaluate the rapidity of a broadcast scheme.

4.2. Simulation Results. The performances of three schemes are compared in terms of reliability, efficiency, and rapidity, respectively.

4.2.1. Reliability. All the three compared schemes show a $99 \%$ or higher reception rate in all beacon cycles of the congestion and smooth scenarios. VBURL, ABSM, and ReC ensure broadcast reliability by performing rebroadcast whenever a vehicle that has not received the message is found. The reception rate does not reach $100 \%$, though, because there are vehicles moving to exit the road on each edge of the target area and exit the simulation network before receiving the message. Because these vehicles are moving farther away from the accident point, there is no safety problem even if they do not receive the message. It is only that they could not take the helpful role to forward safety messages to vehicles beyond the target area.

4.2.2. Broadcasting Efficiency. Figure 9 shows the ratio of vehicles that receive the message against the broadcast count, which signifies the efficiency of a broadcast scheme. The efficiency of VBURL is 2-100 times higher than that of the ABSM and $\mathrm{ReC}$. In particular, the difference is greater in the congestion scenario where the loss of the beacons and traffic concentration are more significant. The beacon inaccuracy caused by the loss of the beacons aggravates the performance of ABSM and $\mathrm{ReC}$ whose forwarding vehicle is determined based on the location information provided by the beacons. On the other hand, the performance of VBURL is intact regardless of the loss of beacons in the congestion scenario since it mainly uses the real-time or semistatic information in its forwarding decision. Furthermore, the efficiency of VBURL even becomes better in the congestion scenario because the number of neighboring vehicles included in the transmission range of a forwarding vehicle is larger.

The efficiency of VBURL decreases slightly as the beacon exchange cycle is lengthened in both of the scenarios. It is because, for the intersection broadcast, VBURL also utilizes holding information from the beacons to determine the roads on which neighboring vehicles exist. As the beacon cycle increases, the beacon information holding time also increases, and, as a result, a road with no neighboring vehicle anymore actually tends not to be recognized early enough, causing unnecessary rebroadcasts. Furthermore, for higher vehicle speed, the information accuracy becomes more sensitive to the beacon cycle. Thus, the broadcast efficiency 
TABLE 2: Number of broadcasts due to the reception of a safety message (A) and the number of broadcasts due to the discovery of a vehicle with no message by a beacon (B).

(a) Congestion scenario

\begin{tabular}{|c|c|c|c|c|c|c|}
\hline \multirow{2}{*}{ Beacon cycle } & \multicolumn{2}{|c|}{ VBURL } & \multicolumn{2}{|c|}{ ABSM } & \multicolumn{2}{|c|}{$\mathrm{ReC}$} \\
\hline & (A) & (B) & (A) & (B) & (A) & (B) \\
\hline 0.5 & 175.6 & 9.7 & 192.1 & 21148.1 & 573.9 & 9321.7 \\
\hline 1.0 & 190.5 & 8.9 & 219.5 & 21727.2 & 371.8 & 1854.8 \\
\hline 3.0 & 181.8 & 7.3 & 267.4 & 24039.6 & 796.7 & 1581.6 \\
\hline 5.0 & 182.2 & 7.9 & 271.7 & 16707.0 & 751.6 & 875.3 \\
\hline 7.0 & 193.7 & 8.3 & 305.4 & 11911.1 & 757.0 & 674.0 \\
\hline 10.0 & 206.3 & 10.5 & 376.6 & 10384.6 & 754.3 & 346.8 \\
\hline
\end{tabular}

(b) Smooth scenario

\begin{tabular}{|c|c|c|c|c|c|c|}
\hline \multirow{2}{*}{ Beacon cycle } & \multicolumn{2}{|c|}{ VBURL } & \multicolumn{2}{|c|}{ ABSM } & \multicolumn{2}{|c|}{$\mathrm{ReC}$} \\
\hline & (A) & (B) & (A) & (B) & (A) & (B) \\
\hline 0.5 & 155.1 & 3.6 & 174.8 & 2434.8 & 334.5 & 1692.7 \\
\hline 1.0 & 157.7 & 3.1 & 190.2 & 2979.2 & 253.9 & 299.2 \\
\hline 3.0 & 166.8 & 4 & 217.1 & 1831.2 & 227.5 & 135.4 \\
\hline 5.0 & 175.2 & 4 & 249.3 & 1544.4 & 244.8 & 119.5 \\
\hline 7.0 & 166.6 & 5.1 & 260.4 & 1318.0 & 287.1 & 97.3 \\
\hline 10.0 & 194.8 & 5.4 & 271.5 & 1344.3 & 451.3 & 95.0 \\
\hline
\end{tabular}
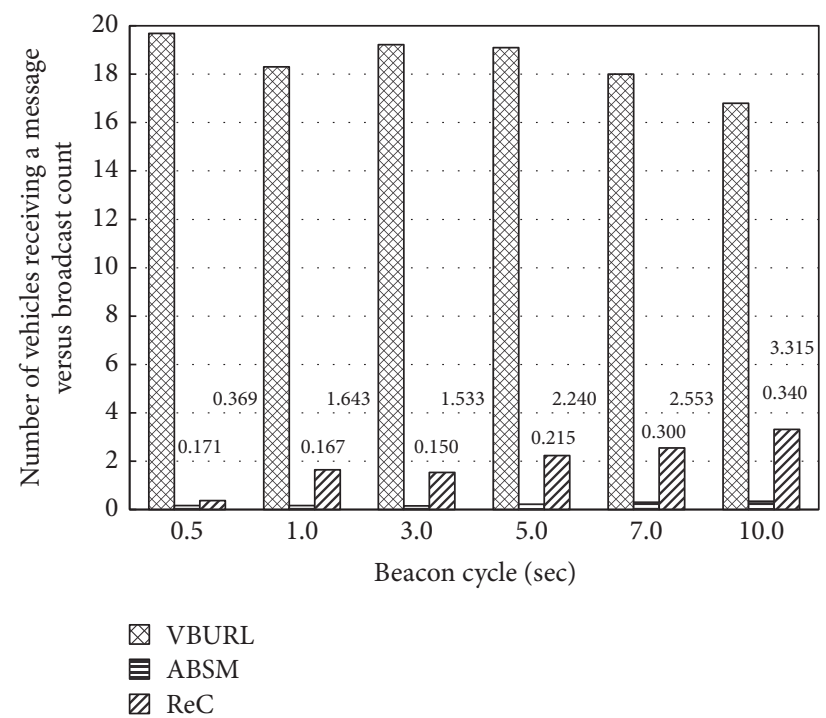

(a) Congestion scenario
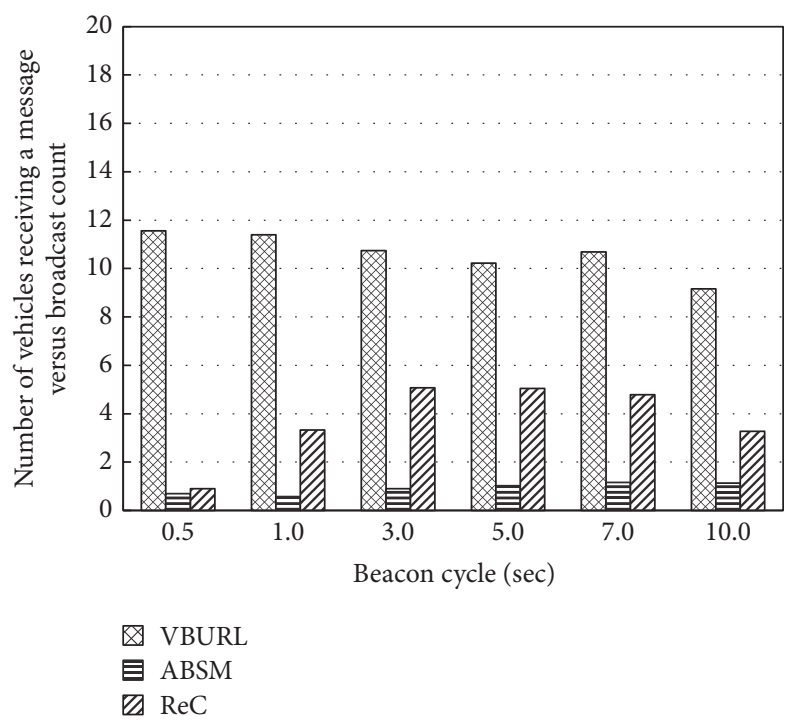

(b) Smooth scenario

Figure 9: Broadcast efficiency.

decreases slightly in the $7 \mathrm{sec}$ or longer beacon cycles in the congestion scenario and in $3 \mathrm{sec}$ or longer beacon cycles in the smooth scenario in VBURL.

ABSM has the lowest efficiency among the compared schemes due to its great inefficiency in the broadcast when a new neighbor with no message is found as shown in Table 2. In the case of ABSM, the efficiency increases slightly in both congestion and smooth scenarios as the beacon cycle lengthens since the efficiency of ABSM is mainly determined by the efficiency of rebroadcast upon the discovery of with-nomessage neighbors, for which the efficiency is better with the longer beacon cycle as shown in Table 2. On the other hand, in the case of $\mathrm{ReC}$, as the beacon cycle becomes longer, the efficiency increases in the congestion scenario whereas the efficiency increases first and then decreases in the smooth scenario. It is because, in $\mathrm{ReC}$, the effect of an efficiency decrement due to the inaccuracy of location information resulting from the long beacon cycle is more prominent in the smooth scenario where the difference in beacon accuracy by the beacon cycle is greater due to the faster speed of vehicles.

Specifically, the message broadcast is attempted when (1) the vehicle receives a new safety message or (2) neighboring 
vehicles with no message are detected by the beacons. Table 2 shows the number of broadcasts occurred during the entire simulation according to by which incident the broadcast is attempted. In VBURL, the least number of broadcasts are incurred on both of the incident (1) and (2) in all beacon cycles of the congestion and smooth scenarios. In particular, In VBURL, the broadcasts are performed mainly by receiving a new safety message. On the contrary, in ReC, more broadcasts are incurred by the detection of a vehicle with no message for relatively short beacon cycles. Furthermore, in ABSM even for all lengths of beacon cycles most of the broadcasts are caused by the incident (2).

The difference between VBURL and the two compared legacy schemes is particularly prominent in (2). Due to the characteristics of urban roads, vehicles with a message and vehicles with no message often encounter each other in groups. When one of the vehicles in the with-no-message group issues a beacon, multiple vehicles in the with-themessage group may receive this beacon and the process to determine the next forwarding vehicle starts immediately. In case of ABSM, all CDS (similarly all non-CDS) vehicles with the message are assigned the same broadcast priority upon receiving the beacon and perform the broadcast simultaneously resulting in broadcast collisions. Moreover, since the beacon generation times of vehicles are not synchronized, in the worst case, whenever a beacon is issued from a vehicle in the with-no-message group, the vehicles in the withthe-message group may attempt to broadcast and cause a collision. In $\mathrm{ReC}$, broadcast priority is assigned in the order of proximity to the vehicle with no message, instead of simply reflecting the number of neighbors with no message as with ABSM (which is one for all the vehicles in the with-themessage group that hears the beacon from a with-no-message vehicle). Therefore, $\mathrm{ReC}$ has a lower possibility of the same broadcast priority among the vehicles in with-the-message group. However, a similar problem to ABSM may still occur due to the inaccuracy of beacon information caused by the loss of a beacon due to collisions in traffic concentration.

The size of a vehicle group tends to be larger in an environment with high vehicle density and worsens the situation. Thus, in VBURL, ABSM, and ReC, the number of broadcasts due to the discovery of a vehicle with no message in the congestion scenario is approximately 2 times, 9.2 times, and 2.1 times larger than in the smooth scenario, respectively. Both ABSM and $\mathrm{ReC}$ have a decreased chance of performing broadcast by discovering the vehicles with no message as the beacon cycle is lengthened. As a result, the number of broadcasts owing to the discovery of vehicles with no message decreases as the beacon cycle gets longer in Table 2 .

VBURL incurs the smallest number of broadcasts resulting from the reception of a safety message even though the difference among the schemes is not as large as the difference in terms of the number of broadcasts resulting from the discovery of vehicles with no message. In all three schemes, the number of broadcasts resulting from the reception of a safety message is around 1.1-1.5 times larger in congestion scenario than in the smooth scenario. It is because the loss of a beacon due to a beacon collision causes the inaccuracy of beacon information in the environment with high vehicle density resulting in decision of suboptimal forwarding vehicle and/or rebroadcast. The performance difference depending on the traffic density scenario is the least in VBURL though since it is the least dependent on the holding information obtained from beacons; that is, the utilization of holding information is limited to the rebroadcast decision at the intersections.

In VBURL, the number of broadcast attempts upon receiving a new safety message slightly increases as the beacon cycle is lengthened. It is because, as the beacon cycle lengthens, a road with no neighboring vehicle anymore actually tends not to be recognized early enough, causing unnecessary rebroadcasts.

The number of broadcasts by the reception of a new safety message is the largest in $\mathrm{ReC}$, which is the most dependent on the accuracy of location information provided by beacons. In $\mathrm{ReC}$, if the beacon cycle is too short, not only the loss of the beacons due to collisions increases, but also more frequent broadcasts occur because the broadcast waiting time becomes shorter (note that the maximum waiting time is set as the beacon cycle in $\mathrm{ReC}$ ). Note that difference in waiting time among the vehicles as well as the length of waiting time becomes smaller as the maximum waiting time becomes shorter and vehicle density becomes higher, leading to higher chance of unnecessary broadcasts. On the other hand, when the beacon cycle becomes too long, not only the inaccuracy of location information obtained from beacon increases, but also the number of vehicles considered as neighbors increases, both of which lead to the degradation in efficiency. As a result, in $\mathrm{ReC}$, the number of broadcasts initiated by the reception of a new safety message first decreases as the beacon cycle became longer, then it starts to increase when the beacon cycle rises above a certain value.

In the case of ABSM, the number of broadcast attempts upon receiving a new safety message increases as the beacon cycle is lengthened. For ABSM, not only the beacon inaccuracy increases as the beacon cycle is lengthened but also the average number of neighboring vehicles tends to increase due to the increase in beacon hold time. If the number of vehicles regarded as neighbors increases, the broadcast waiting time becomes shorter and tends to be similar among vehicles in ABSM, thus increasing the chance of unnecessary broadcasts or collisions.

4.2.3. Latency. Figure 10 shows the transient behavior in the changing ratio of vehicles receiving the message over time in the congestion and smooth scenarios, respectively. In both congestion and smooth scenarios of all the three schemes, at least $99 \%$ of vehicles receive the message after an interval that is slightly longer than the traffic signal waiting time after the first broadcast. It is because when one interval of signal waiting time passes after the first broadcast, it is highly likely that a vehicle with a message has passed every intersection. As a result, the possibility of message dissemination to most of the roads in the simulation network is high.

The percentage of vehicles receiving a message within one second after the first broadcast in the smooth scenario (Figures 10(d), 10(e), and 10(f)) is lower than in the congestion scenario (Figures 10(a), 10(b), and 10(c)). It is because the vehicle density of the smooth scenario is lower than that of the 


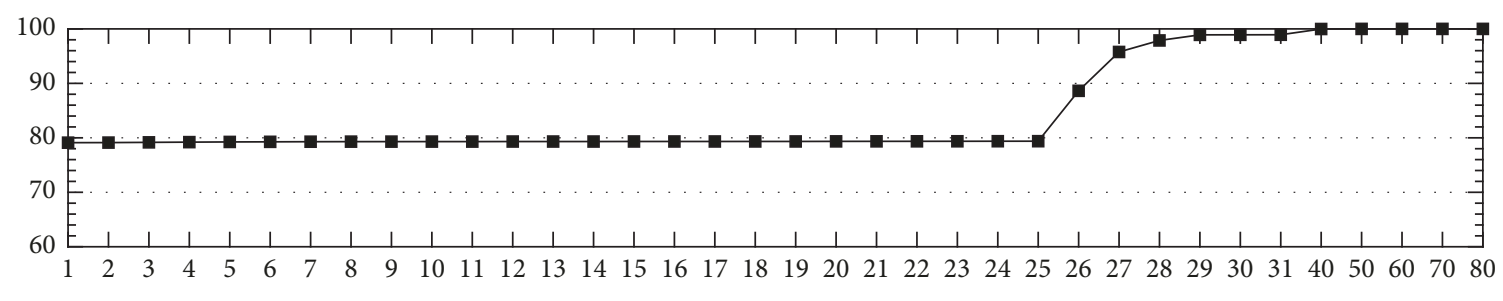

$\rightarrow 0.5$

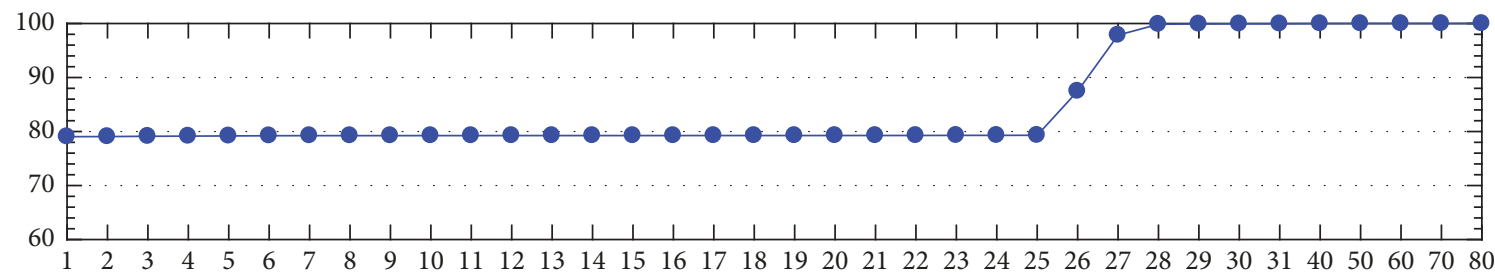

$\rightarrow 1.0$

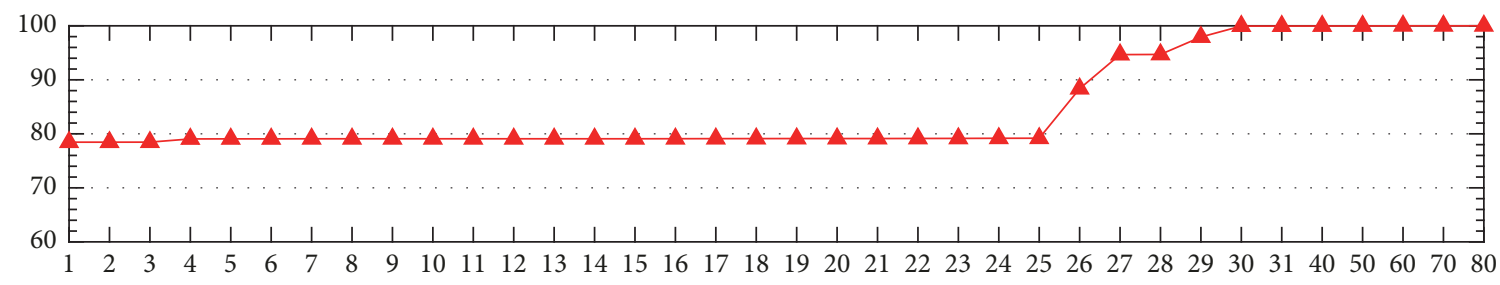

$-3.0$

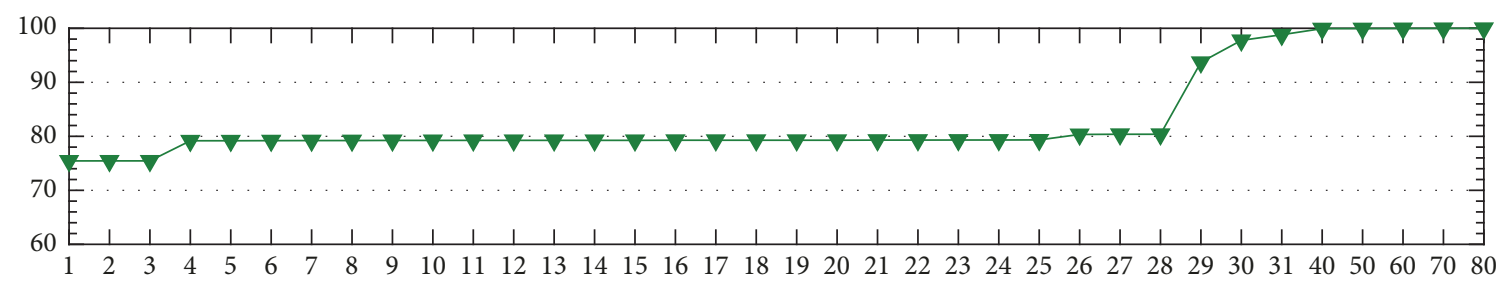

$\rightarrow 5.0$

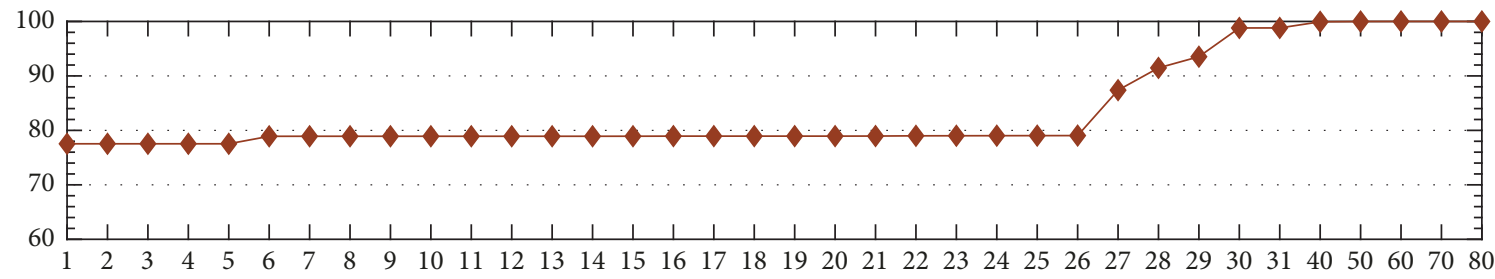

$\bullet 7.0$

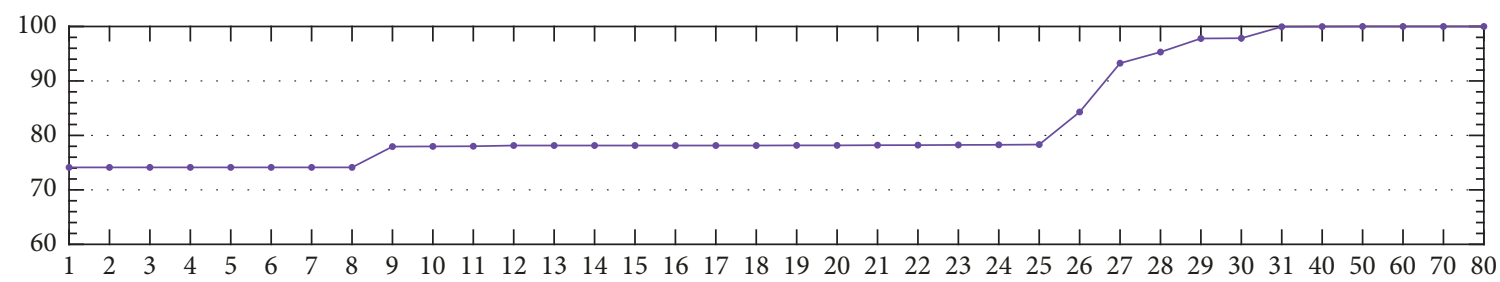

(a) Congestion scenarios: VBURL ( $x$ axis: time (sec), $y$ axis: percentage of vehicle receiving the message)

Figure 10: Continued. 


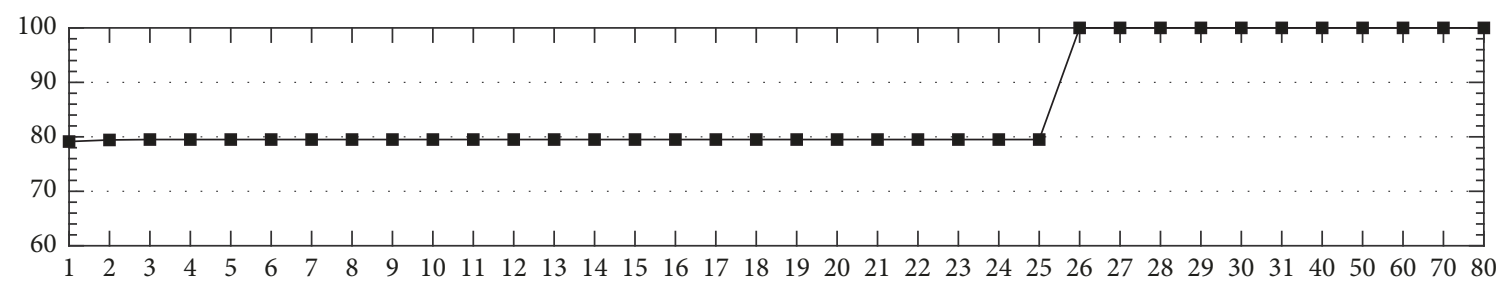

$\rightarrow-0.5$

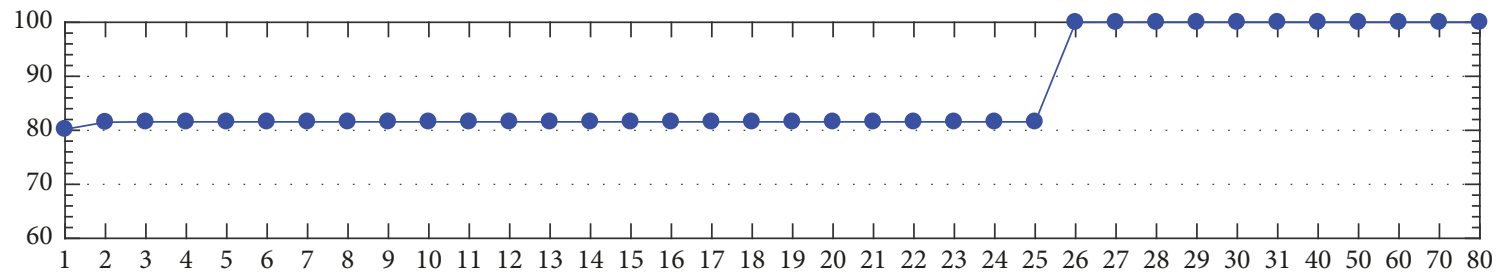

$-1.0$

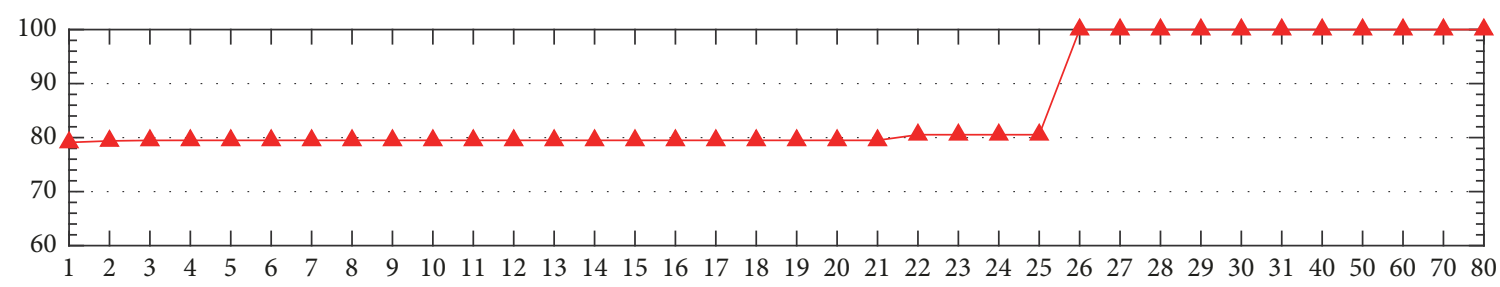

$-3.0$

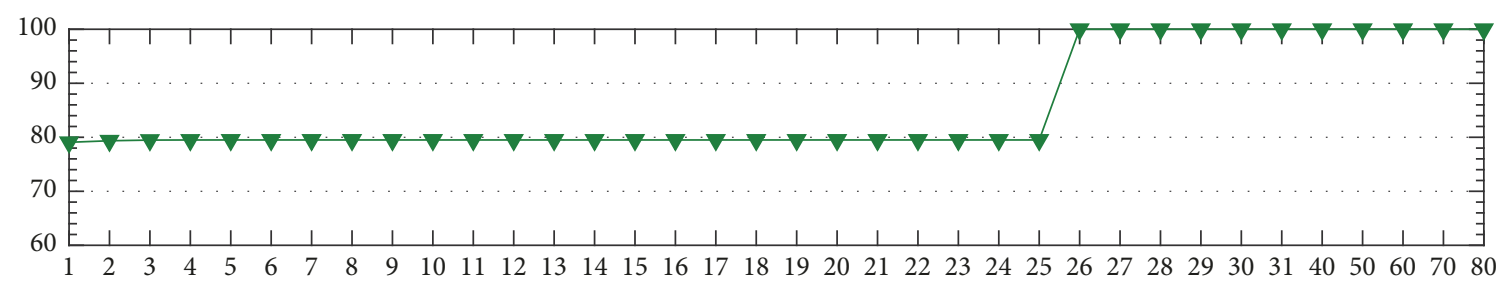

$\neg-5.0$

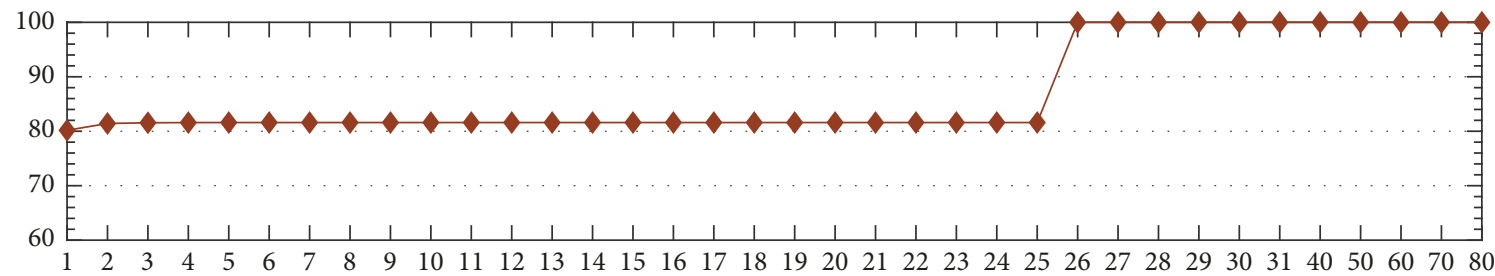

$-7.0$

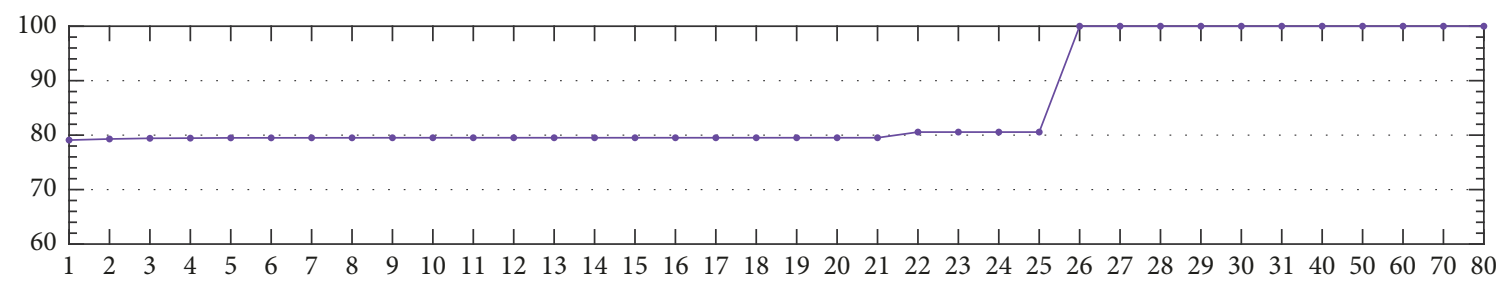

(b) Congestion scenarios: ABSM ( $x$ axis: time (sec), $y$ axis: percentage of vehicle receiving the message)

Figure 10: Continued. 


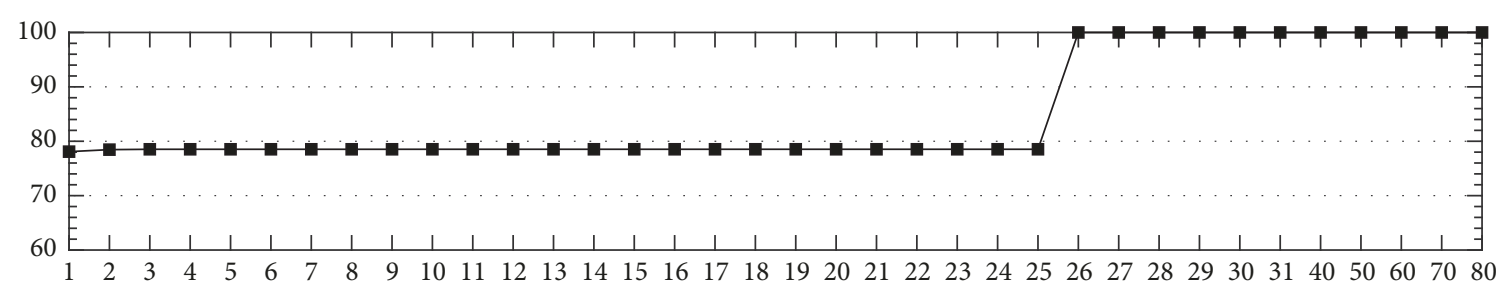

$\rightarrow-0.5$

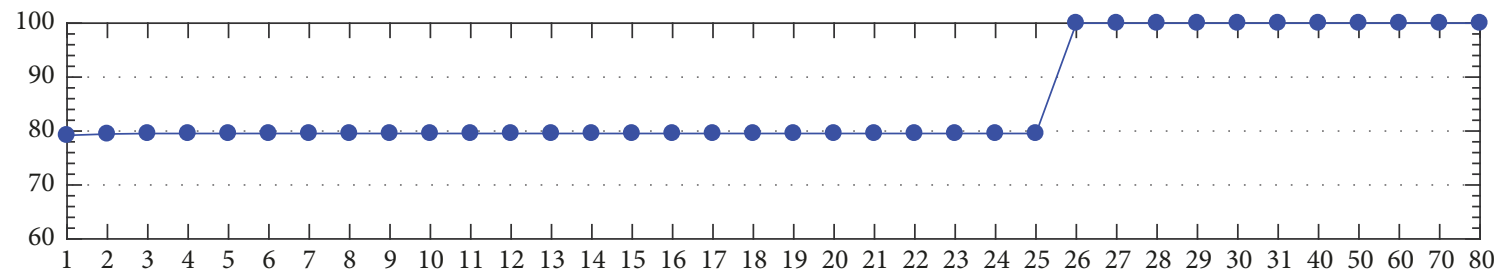

$\longrightarrow 1.0$

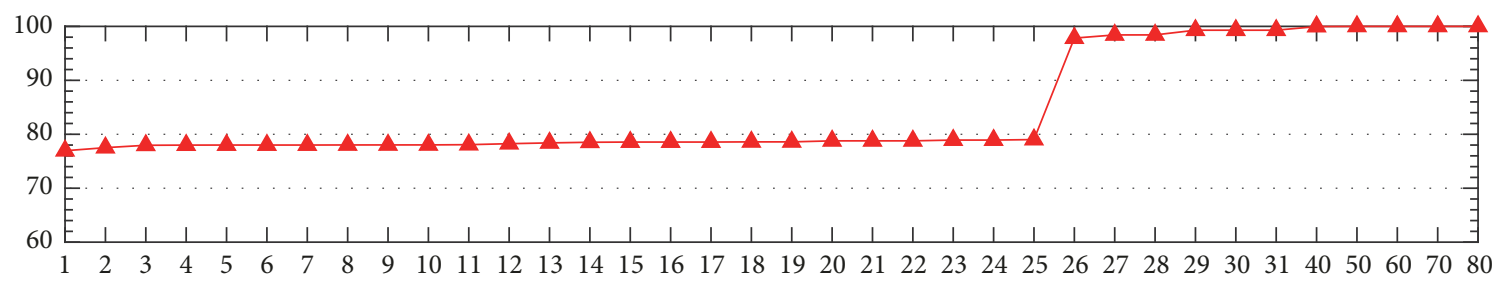

$-3.0$

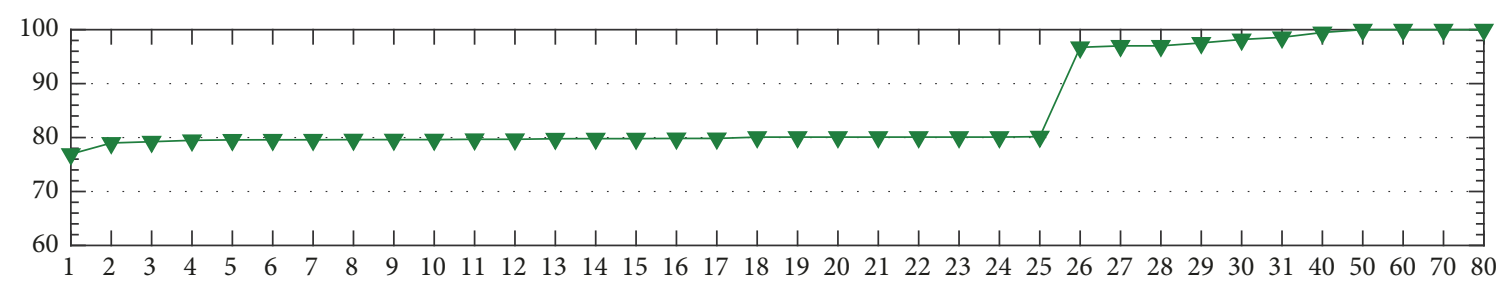

$\neg 5.0$

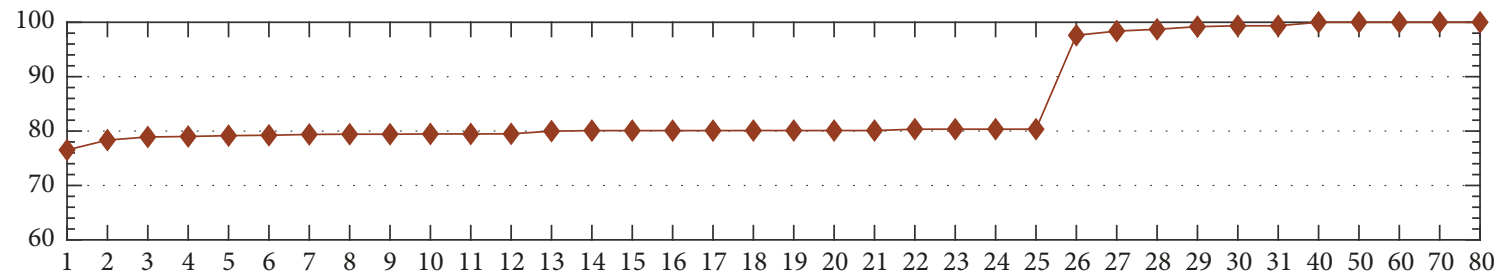

$\checkmark 7.0$

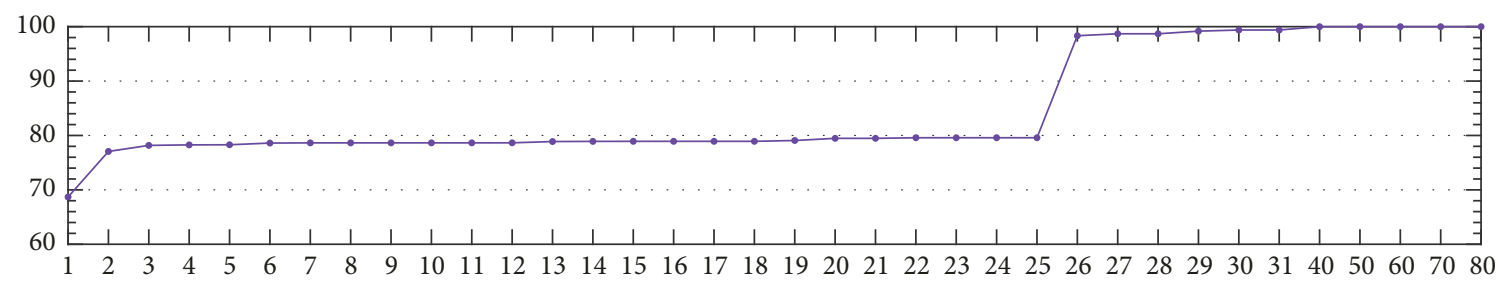

(c) Congestion scenarios: $\operatorname{ReC}(x$ axis: time (sec), $y$ axis: percentage of vehicle receiving the message)

Figure 10: Continued. 

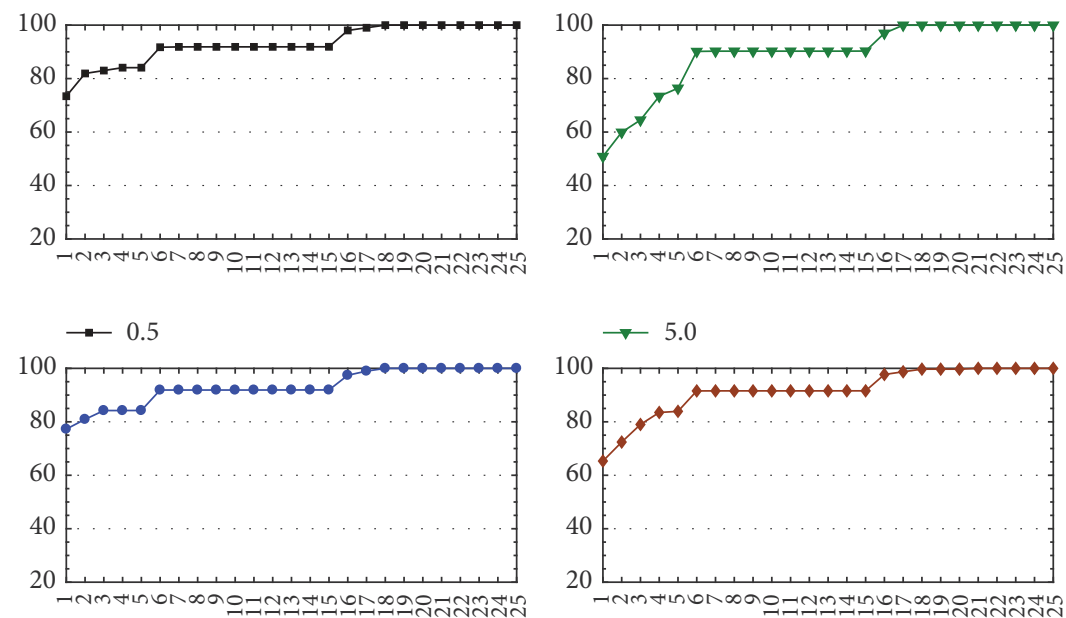

$\rightarrow 5.0$
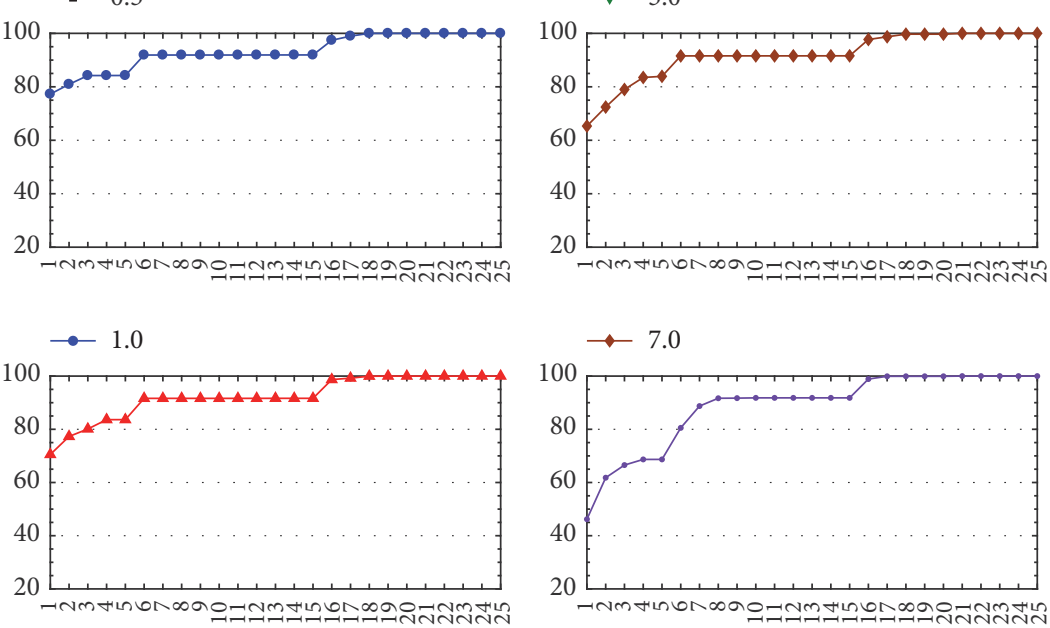

$\rightarrow 3.0$

$\rightarrow 10.0$

(d) Smooth scenarios: VBURL ( $x$ axis: time (sec), $y$ axis: percentage of vehicle receiving the message)
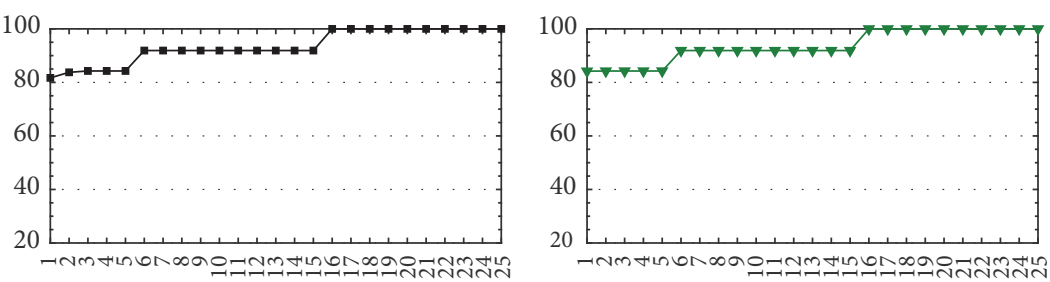

$\rightarrow 0.5$
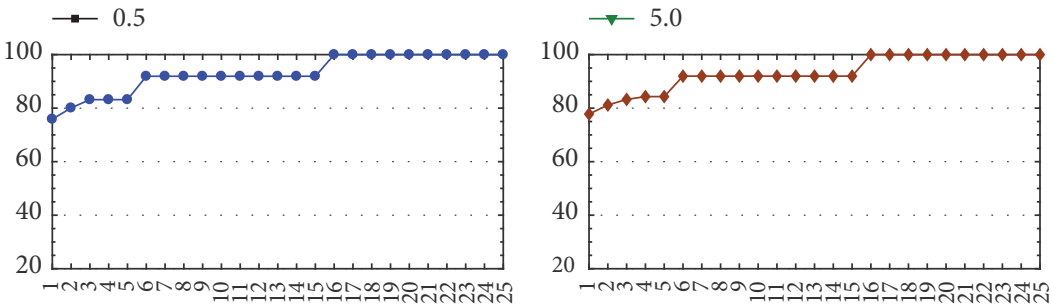

$-1.0$

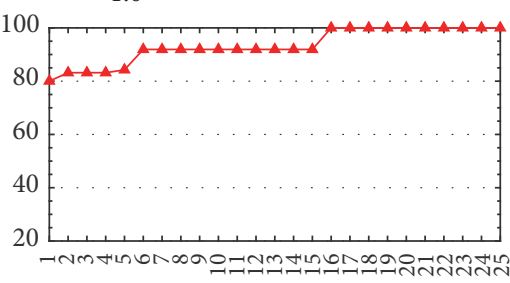

$-7.0$

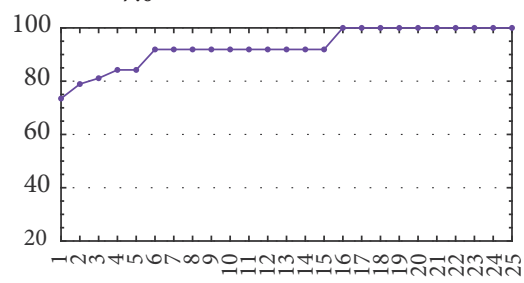

$\simeq 3.0$

$\rightarrow 10.0$

(e) Smooth scenarios: ABSM ( $x$ axis: time (sec), $y$ axis: percentage of vehicle receiving the message)

Figure 10: Continued. 

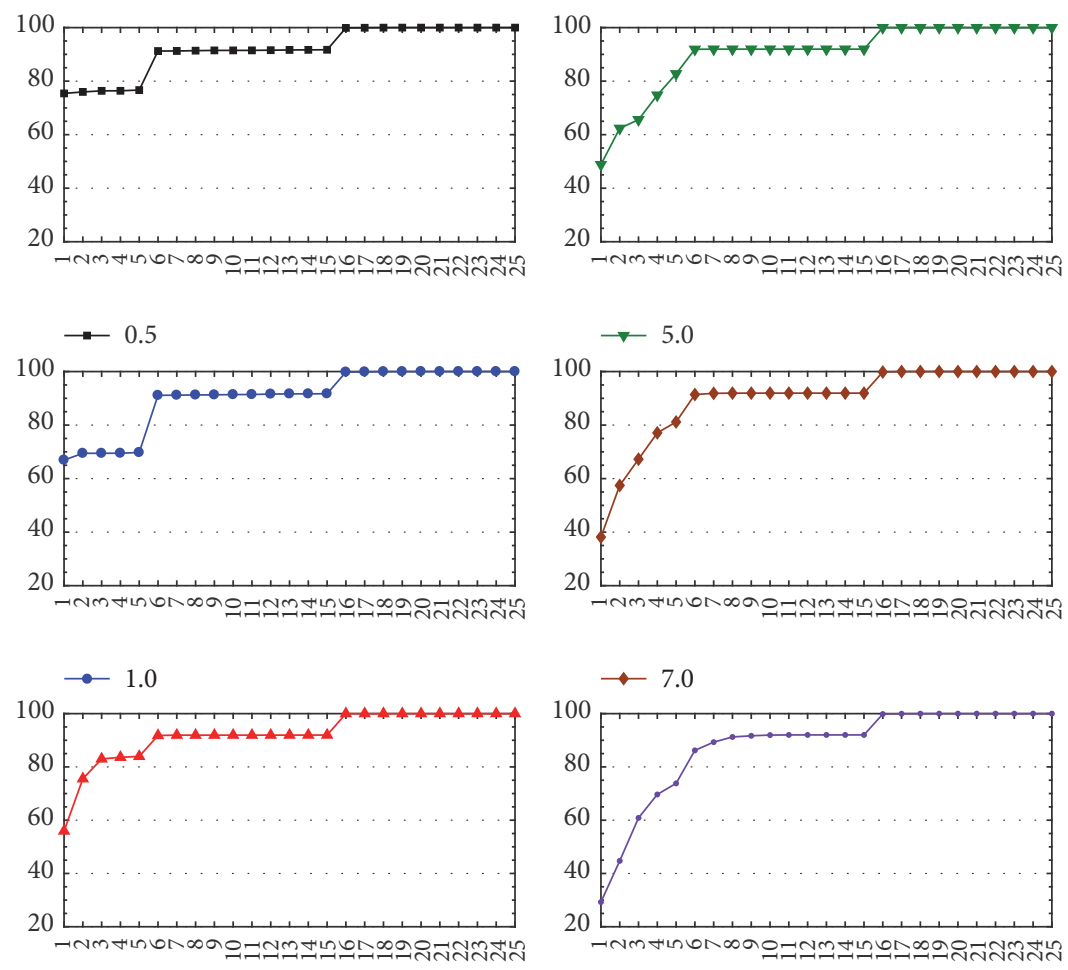

$\triangle 3.0$

$\rightarrow 10.0$

(f) Smooth scenarios: $\operatorname{ReC}(x$ axis: time (sec), $y$ axis: percentage of vehicle receiving the message)

FIGURE 10: Delay of message reception.

congestion scenario and has a higher possibility of suspension of message dissemination. The suspended broadcast could be resumed when a vehicle with no message is discovered by a vehicle with the message through a beacon, and the time required to discover a vehicle with no message tends to increase as the beacon cycle becomes longer. Thus, in all of the three compared schemes, the percentage of vehicles receiving a message within one second tends to decrease as the beacon cycle is lengthened.

Meanwhile, ABSM has a slightly higher percentage of message reception within one second compared to VBURL and ReC. This is not only because waiting time of ABSM tends to be shorter than the other two schemes, but also at the cost of very low efficiency and large number of broadcasts as shown in Figure 9 and Table 2. For the percentage of message reception within one second, $\mathrm{ReC}$ is the lowest in all cycles except for the case of $0.5 \mathrm{sec}$ beacon cycle. The reason for this is that as the beacon cycle becomes longer, beacon inaccuracy becomes greater, causing as a result more broadcast collisions or longer delay due to no forwarding vehicle with the first priority.

In the congestion scenario (Figures 10(a), 10(b), and 10(c)), all vehicles in ABSM receive messages before 30 seconds, while VBURL and $\mathrm{ReC}$ have few vehicles that receive messages after 30 seconds. For the vehicles whose delay of message reception is more than 30 seconds in VBURL, (1) the distance to the accident point when the message is first issued, (2) the ratio of the distance travelled before reception of message and the original distance when the message is first issued, and (3) the message reception delay is measured in order to check how critical the delay could be to the quality of application. As shown in Figure 11, the vehicles with longer delay of reception tend to be farther away from the accident point when the message is first issued. Even though it takes longer than 30 seconds to receive the message, they receive it at least one intersection ahead of the accident point and, on average, around three or more intersections away from the accident point, that is, way ahead of reaching the accident point. Since they were far from the accident, taking longer time in receiving the message is not critical to the purpose of application. Even in the case where the reception delay is long, the message is received with a sufficient distance to be able to respond to the accident by decelerating or rerouting to a bypass. In our experiments, the vehicle that receives a message the latest receives the message at 77.5 seconds after the broadcast starts, and it is still around 3.8 intersections away from the accident point when the message is received.

Figure 12 shows the average message reception delay for varying beacon cycles. In all cases, the difference in average reception time among VBURL, ABSM, and $\mathrm{ReC}$ is less than 1.5 seconds, and the movement distance corresponding to this time is $8-21 \mathrm{~m}$ depending on the vehicle speed. Therefore, it does not result in a significant difference that would affect the safety service quality on urban roads where the vehicle density and congestion are high and the speed is low. The factors that have the greatest effect on the average reception 


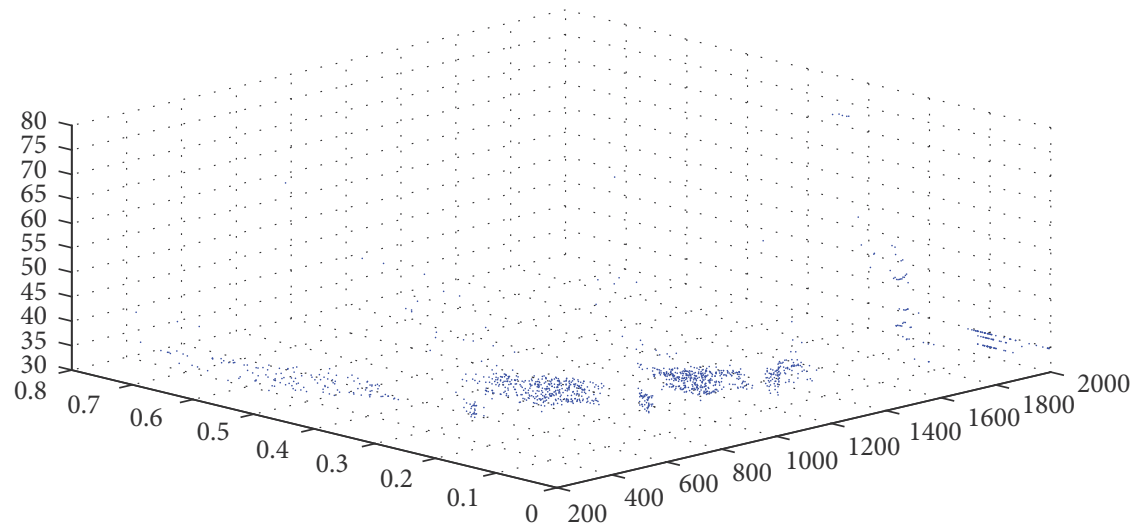

FIGURE 11: Safety message delivery QoS for vehicles receiving messages 30 seconds after the first broadcast.

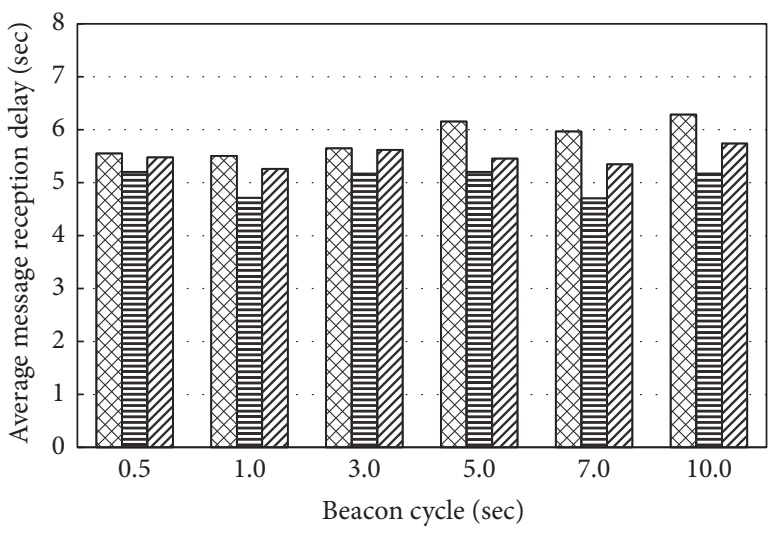

$$
\begin{aligned}
& \text { 冈BURL } \\
& \text { 目 ABSM } \\
& \text { \eC }
\end{aligned}
$$

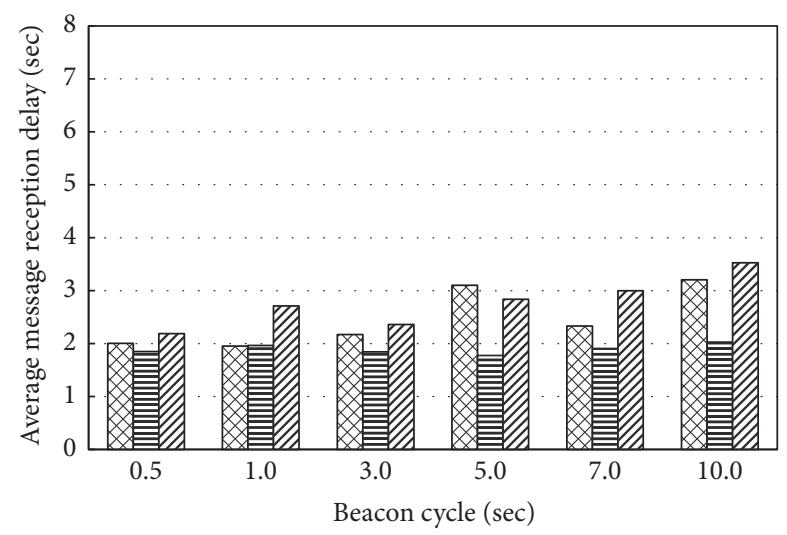

$$
\begin{aligned}
& \text { 林URL } \\
& \text { 目 ABSM } \\
& \text { ש ReC }
\end{aligned}
$$

(a) Congestion scenario

(b) Smooth scenario

Figure 12: Average message reception delay.

delay are length of traffic signals and vehicle speed. The average reception delay of the congestion scenario $(5-6 \mathrm{sec})$, where traffic signal cycle is longer and vehicle speed is slow due to high vehicle density and traffic congestion, is longer by $2.8-3.4 \mathrm{sec}$ than in the smooth scenario $(2-3 \mathrm{sec})$.

VBURL and ReC show a trend of slightly increasing average reception delay as the beacon cycle is lengthened, because it takes longer to discover a vehicle with no message. According to the way how the broadcast waiting time is set, VBURL tends to have a longer broadcast waiting time than $\mathrm{ABSM}$ and ReC. As a result, in most of the cases, the average reception delay of VBURL is slightly longer than those of the compared schemes. In the case of ReC, the message reception delay tends to be higher when the vehicle density is high and the beacon cycle is long. It is because the information inaccuracy increases as the vehicle density and/or the beacon cycle increase, and as a result, multiple forwarding vehicles with the highest priority may exist resulting in collisions or broadcast delay increases due to the lack of a vehicle with the highest priority. In the smooth scenario, where the vehicle speed is high and the inaccuracy of location information tends to be higher, there are cases where the average reception delay of $\mathrm{ReC}$ is longer than that of VBURL. In the case of ABSM, the average reception delay is the shortest among the compared schemes for all beacon cycles. The waiting time of ABSM tends to be very short due to too many rebroadcasts meaning extremely low efficiency, as shown in Figure 9 and Table 2 .

\section{Conclusions}

A broadcast message dissemination scheme with minimal beacon dependency is proposed considering the characteristics of urban roads such as frequent intersections, high vehicle density, and traffic concentration due to frequent traffic signals. The proposed VBURL selects the forwarding vehicle only using its location and the location of the current forwarding vehicle which is conveyed in the received message, that is, real-time information, and by leveraging the road layout information which is available from the digital map obtained through the DMB network. On urban roads, the intersections and the roads connecting the intersections are 
repeated. On a straight road, the vehicle that is farthest from the current forwarding vehicle simply performs broadcast forwarding for rapid and efficient broadcast dissemination. In intersections, however, where signal attenuation is more serious due to surrounding obstacles such as buildings, the vehicle located in the intersection area that is free from such influence performs additional broadcasts in order to facilitate broadcast dissemination to all the roads adjacent to the intersection. The beacon only serves a supplementary role to deliver a safety message to the vehicles that have not received the message, which are newly entering an area where the broadcast has been completed. For performance evaluation, the percentage of vehicles receiving messages, the number of vehicles receiving the message per broadcast, and the message reception delay are measured for various beacon cycles in the congestion/smooth scenarios. All of the three compared schemes, VBURL, ABSM, and ReC, show reliabilities close to $100 \%$, but the broadcast efficiency of VBURL is 2-100 times higher than that of ABSM and ReC for all beacon cycles in both of the congestion and smooth scenarios. Difference in efficiency between VBURL and the compared schemes is greater in congestion scenario for which the traffic concentration as well as the loss of beacons is more serious. In particular, the broadcasts inefficiency of ABSM and $\mathrm{ReC}$ is significant when neighboring vehicles with no message are detected. In terms of message propagation speed, the average message reception delay of VBURL is slightly longer than that of ABSM and ReC. The difference in the average reception delay among the three schemes is, though, less than 1.5 seconds, which would not have a significant effect on quality of service. Furthermore, in VBURL, the vehicles whose message reception delay is long are those located far from the accident point at the time of the first broadcast, and all the vehicles receive the safety message early enough to have sufficient chance to reroute to a bypass before reaching the accident point.

\section{Conflicts of Interest}

The authors declare that they have no conflicts of interest.

\section{Acknowledgments}

This work was supported by Institute for Information \& Communications Technology Promotion (IITP) Grant funded by the Korea government (MSIT) (no. 2015-0-00183, “A Study on Hyper Connected Self-Organizing Network Infrastructure Technologies for IoT Service").

\section{References}

[1] IEEE P802.11p/D6.01, "Part 11: Wireless lan medium access control (MAC) and physical layer (phy) specifications-amendment 7: Wireless access in vehicular environments," Apr 2009.

[2] IEEE Std, "IEEE Standard for Wireless Access in Vehicular Environments (WAVE) Multi-Channel Operation," Sep. 2010.

[3] M. Nekovee, "Sensor networks on the road: the promises and challenges of vehicular ad hoc networks and vehicular grids," in Procceedings of the Workshop on Ubiquitous Computing and e-Research, May 2005.

[4] X. Ma, J. Zhang, and T. Wu, "Reliability analysis of one-hop safety-critical broadcast services in VANETs," IEEE Transactions on Vehicular Technology, vol. 60, no. 8, pp. 3933-3946, 2011.

[5] M. M. I. Taha and Y. M. Y. Hasan, "VANET-DSRC protocol for reliable broadcasting of life safety messages," in Proceedings of the ISSPIT 2007 - 2007 IEEE International Symposium on Signal Processing and Information Technology, pp. 104-109, Egypt, December 2007.

[6] Y. Li, H. Zhang, Z. Huang, and M. Albert, "Optimal link scheduling for delay-constrained periodic traffic over unreliable wireless links," in Proceedings of the 33rd IEEE Conference on Computer Communications, IEEE INFOCOM 2014, pp. 14651473, Canada, May 2014.

[7] S. Wang, B. Yang, F. Gu, and X. Shi, "A novel reliable broadcast protocol for VANET's safety applications," in Proceedings of the 6th IEEE International Conference on Electronics Information and Emergency Communication, ICEIEC 2016, pp. 282-286, China, June 2016.

[8] W. Zhu, D. Gao, C. H. Foh, W. Zhao, and H. Zhang, "A collision avoidance mechanism for emergency message broadcast in urban VANET," in Proceedings of the 83rd IEEE Vehicular Technology Conference, VTC Spring 2016, China, May 2016.

[9] M. Koubek, S. Rea, and D. Pesch, "Reliable broadcasting for active safety applications in vehicular highway networks," in Proceedings of the 2010 IEEE 71st Vehicular Technology Conference, VTC 2010-Spring, Taiwan, May 2010.

[10] C. Wu, K. Kumekawa, and T. Kato, "A novel multi-hop broadcast protocol for vehicular safety applications," Journal of Information Processing, vol. 18, pp. 110-124, 2010.

[11] C. Liu and C. Chigan, "RPB-MD: providing robust message dissemination for vehicular ad hoc networks," Ad Hoc Networks, vol. 10, no. 3, pp. 497-511, 2012.

[12] S. Biswas, R. Tatchikou, and F. Dion, "Vehicle-to-vehicle wireless communication protocols for enhancing highway traffic safety," IEEE Communications Magazine, vol. 44, no. 1, pp. 7482, 2006.

[13] F. Ye, M. Adams, and S. Roy, "V2V wireless communication protocol for rear-end collision avoidance on highways," in Proceedings of the IEEE International Conference on Communications Workshops (ICC '08), pp. 375-379, Beijing, China, May 2008.

[14] G. Xiao, H. Zhang, Z. Huang, and Y. Chen, "Decentralized cooperative piggybacking for reliable broadcast in the VANET," in Proceedings of the 83rd IEEE Vehicular Technology Conference, VTC Spring 2016, China, May 2016.

[15] X. Zhen, J. Wang, P. Wang, X. Wang, and F. Liu, "A senderinitiated adaptive and reliable broadcast scheme for VANET safety message," in Proceedings of the 2012 4th International Symposium on Information Science and Engineering, ISISE 2012, pp. 329-334, China, December 2012.

[16] X. Ma, J. Zhang, X. Yin, and K. S. Trivedi, "Design and analysis of a robust broadcast scheme for VANET safety-related services," IEEE Transactions on Vehicular Technology, vol. 61, no. 1, pp. 46-61, 2012.

[17] N. Wisitpongphan, O. K. Tonguz, J. S. Parikh, P. Mudalige, F. Bai, and V. Sadekar, "Broadcast storm mitigation techniques in vehicular ad hoc networks," IEEE Wireless Communications Magazine, vol. 14, no. 6, pp. 84-94, 2007.

[18] G. Korkmaz, E. Ekici, and F. Ozguner, "Black-burst-based multihop broadcast protocols for vehicular networks," IEEE 
Transactions on Vehicular Technology, vol. 56, no. 5, pp. 31593167, 2007.

[19] Y. Bi, H. Shan, X. S. Shen, N. Wang, and H. Zhao, "A multihop broadcast protocol for emergency message dissemination in urban vehicular ad hoc networks," IEEE Transactions on Intelligent Transportation Systems, vol. 17, no. 3, pp. 736-750, 2016.

[20] F. J. Ros, P. M. Ruiz, and I. Stojmenovic, "Acknowledgmentbased broadcast protocol for reliable and efficient data dissemination in vehicular ad hoc networks," IEEE Transactions on Mobile Computing, vol. 11, no. 1, pp. 33-46, 2012.

[21] J. Liu, Z. Yang, and I. Stojmenovic, "Receiver consensus: ontime warning delivery for vehicular ad-hoc networks," IEEE Transactions on Emerging Topics in Computing, vol. 1, no. 1, pp. 57-68, 2013.

[22] Y. Sung and M. Lee, "Light-weight reliable broadcast message delivery for vehicular ad-hoc networks," in Proceedings of the IEEE 75th Vehicular Technology Conference, VTC Spring 2012, Japan, June 2012.

[23] K. Obara, R. Akamatsu, and H. Shigeno, "BRNT: Broadcast protocol with road network topology for urban Vehicular Ad Hoc Networks," in Proceedings of the 82nd IEEE Vehicular Technology Conference, VTC Fall 2015, USA, September 2015.

[24] S. Eichler, "Performance evaluation of the IEEE 802.11 p WAVE communication standard," in Proceedings of the 66th IEEE Vehicular Technology Conference (VTC '07-Fall), pp. 2199-2203, Baltimore, MD, USA, October 2007.

[25] V. Nguyen, T. Z. Oo, P. Chuan, and C. S. Hong, "An efficient time slot acquisition on the hybrid TDMA/CSMA multichannel MAC in VANETs," IEEE Communications Letters, vol. 20, no. 5, pp. 970-973, 2016.

[26] C.-Y. Chang, H.-C. Yen, and D.-J. Deng, "V2V QoS guaranteed channel access in IEEE 802.11p VANETs," IEEE Transactions on Dependable and Secure Computing, vol. 13, no. 1, pp. 5-17, 2016.

[27] K. A. Hafeez, A. Anpalagan, and L. Zhao, "Optimizing the control channel interval of the dsrc for vehicular safety applications," IEEE Transactions on Vehicular Technology, vol. 65, no. 5, pp. 3377-3388, 2016.

[28] I. Stojmenovic, M. Seddigh, and J. Zunic, "Dominating sets and neighbor elimination-based broadcasting algorithms in wireless networks," IEEE Transactions on Parallel and Distributed Systems, vol. 13, no. 1, pp. 14-25, 2002.

[29] W. Peng and X. Lu, "On Reducing Broadcast Redundancy in Ad Hoc Wireless Networks," IEEE Trans. Mobile Computing, vol. 1, no. 2, pp. 111-122, 2002.

[30] H. Fübler, J. Widmer, M. Käsemann, M. Mauve, and H. Hartenstein, "Contention-based forwarding for mobile ad hoc networks," Ad Hoc Networks, vol. 1, no. 4, pp. 351-369, 2003, Elsevier.

[31] S. R. Theodore, Wireless Communications: Principles and Practice, Prentice Hall, 2nd edition, 2004. 


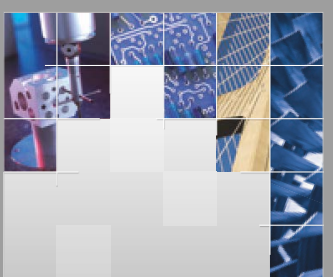

\section{Enfincering}
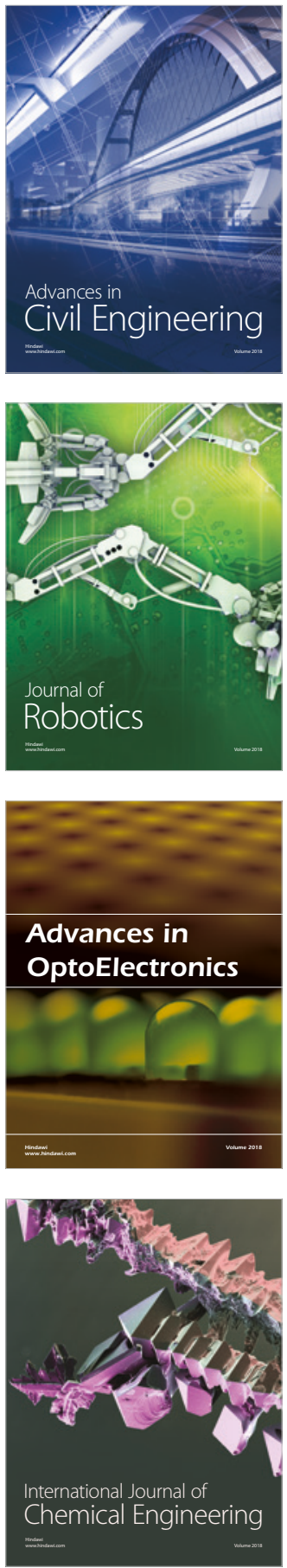

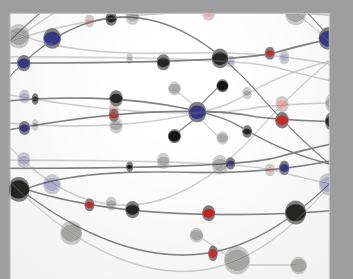

\section{Rotating \\ Machinery}

The Scientific World Journal

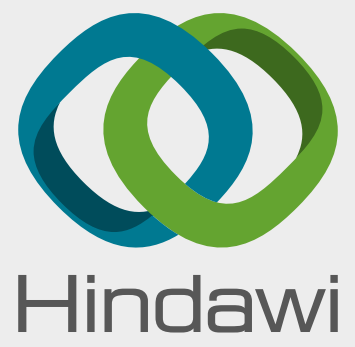

Submit your manuscripts at

www.hindawi.com
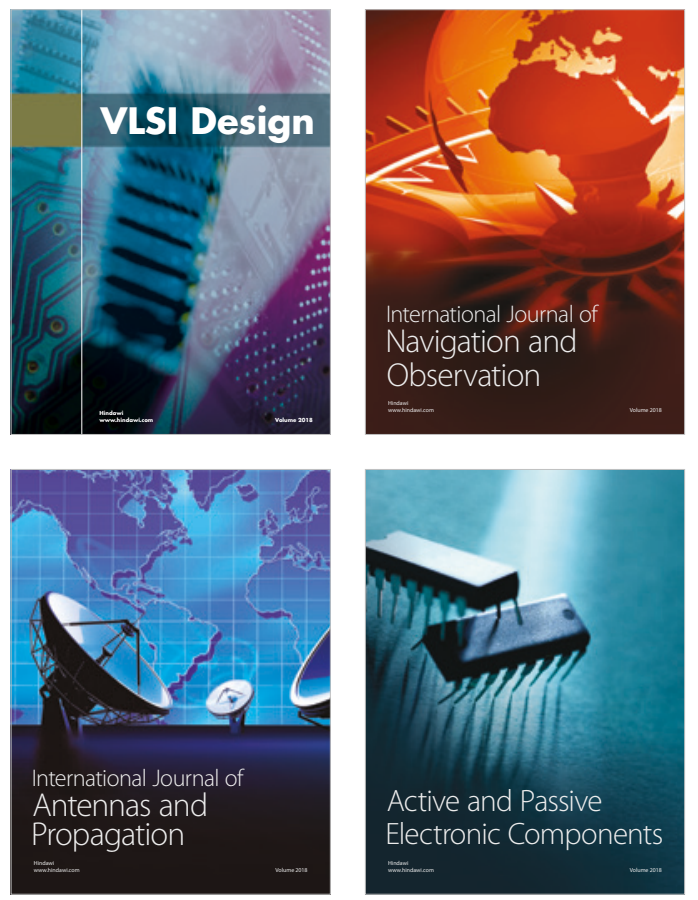
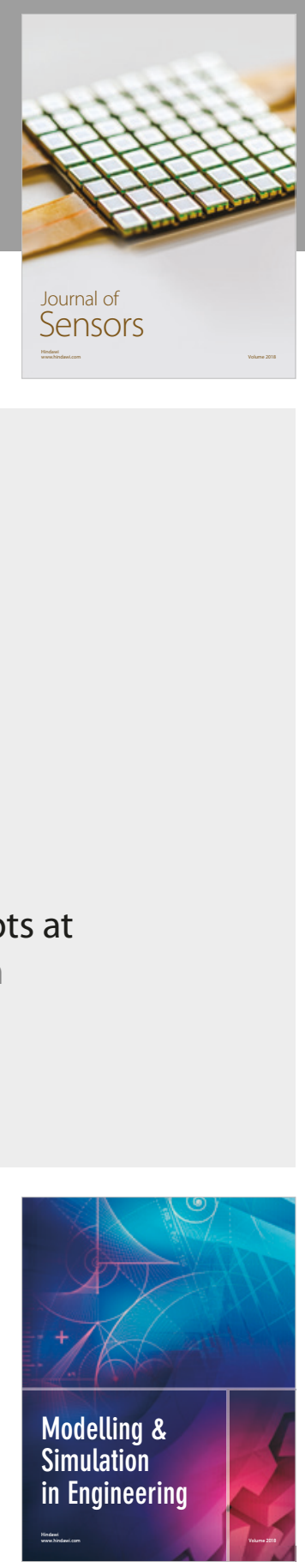

\section{Advances \\ Multimedia}
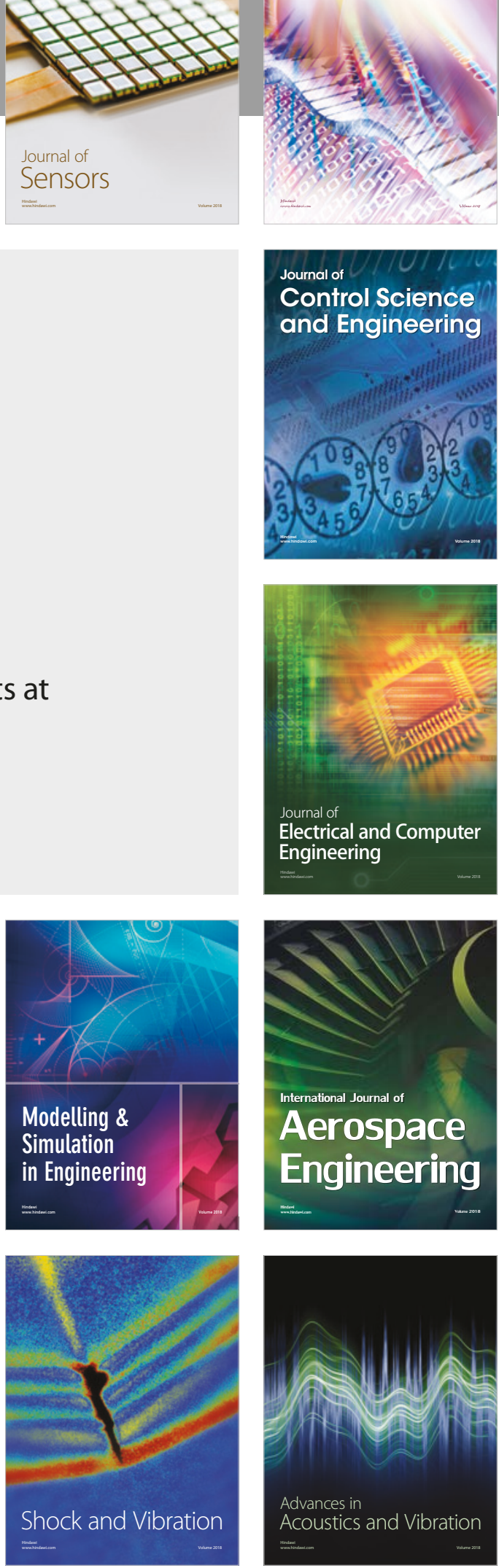